(C) 2018. This manuscript version is made available under the CC-BY-NC-ND 4.0 license http:// creativecommons.org/licenses/by-nc-nd/4.0/ 


\title{
A laboratory experiment on the heuristic switching model*
}

\author{
Mikhail Anufriev ${ }^{a}, \dagger$ \\ Aleksei Chernulich ${ }^{\mathrm{a}, \ddagger}$ \\ Jan Tuinstrab,§ \\ a University of Technology Sydney, Business School, Economics Discipline Group \\ b CeNDEF, Amsterdam School of Economics, University of Amsterdam
}

\begin{abstract}
We present results from the first laboratory experiment on the seminal heuristic switching model introduced by Brock and Hommes (1997, 1998). Subjects choose between two alternatives, a sophisticated and stabilizing, but costly, heuristic, and a destabilizing, but cheap, heuristic, and are paid according to the performance of the chosen heuristic. Aggregate choices determine the evolution of a state variable and, consequently, the performance of both heuristics. Theoretically, an increase in the costs for the stabilizing heuristic generates instability and leads to endogenous fluctuations in both the state variable and the fraction of agents using that heuristic.

We vary the costs of the stabilizing heuristic in the experiment and find that the predictions of the heuristic switching model are partially confirmed. For low costs the dynamics are stable. For high costs, the dynamics initially are unstable and exhibit the type of bubbles and crashes emblematic for the heuristic switching model. However, over time the pattern of bubbles and crashes disappears and the dynamics become more stable. We estimate a standard discrete choice model on aggregate choice data and observe that
\end{abstract}

*We thank the participants of the Society for the Advancement of Behavioral Economics (SABE) Conference, Newcastle, July 2017, "Computation in Economics and Finance" conference, New York, June 2017, the Symposium for Experimental Economics, Dongbei, September 2017, and seminar participants at the University of Amsterdam, University of Technology Sydney, European University at St. Petersburg, and The National Research University Higher School of Economics for helpful discussions. We benefited from stimulating comments by Te Bao, Cees Diks, Cars Hommes, Dávid Kopányi, and an anonymous referee. Mikhail Anufriev and Jan Tuinstra acknowledge financial support from the Australian Research Council through Discovery Project DP140103501. In addition, Jan Tuinstra acknowledges support from the Open Research Area project on Behavioral and Experimental Analyses in Macro-finance (BEAM).

${ }^{\dagger}$ E-mail: Mikhail.Anufriev@uts.edu.au.

$\ddagger$ E-mail: Aleksei.Chernulich@uts.edu.au.

$\S$ E-mail: J.Tuinstra@uva.nl. 
subjects have a tendency to become less sensitive to payoff differences when the environment is less stable, which has important implications for the application of heuristic switching models.

Keywords: Heuristic switching model, Heterogeneous agent models, Discrete choice, Experiments.

JEL Classification: C25, C91, D83. 


\section{Introduction}

In the last couple of decades heterogeneous agents models have become increasingly popular as a description of turbulence and volatility on financial markets. In these models different types of traders coexist on financial markets, as motivated by early empirical and theoretical studies. Frankel and Froot (1990), for example, distinguish between fundamentalists, who use in-depth analysis of firms and their market environment to determine the fundamental value of an asset and believe that prices tend to revert back to this fundamental value, and chartists, who use technical analysis to identify patterns in prices and extrapolate those when predicting future prices (also see Allen and Taylor, 1990). Day and Huang (1990) and Chiarella (1992) show that the interaction between these different types of traders may lead to the emergence of endogenous fluctuations in asset prices in an otherwise stationary and deterministic environment.

An important class of heterogeneous agents models assumes that there is a large population of traders that adaptively switch between some archetypal types of behavior, or heuristics, on the basis of the relative performance of these heuristics, see, e.g., Brock and Hommes (1997, 1998), Chiarella and He (2002, 2003) and Anufriev and Hommes (2012a,b). These models, often referred to as heuristic switching models, have been successful in describing stylized facts of financial markets, such as bubbles and crashes in asset prices, excess volatility, volatility clustering, and fat tails, and have become quite popular as a result. The most common approach of modeling switching between heuristics in this literature is through the so-called discrete choice model. A crucial role in that model is played by the Intensity of Choice (IoC) parameter, which measures how sensitive traders are with respect to differences in performance. Brock and Hommes $(1997,1998)$ showed, and follow-up research confirmed, that heuristic switching models generate excess volatility and many other stylized facts of financial markets when the IoC parameter is sufficiently large. The IoC parameter is thus pivotal for the dynamic properties of the heuristic switching models, and hence for their success in explaining financial market data and for the validity of policy implications.

In this paper we present the results of a laboratory experiment that is designed to test the predictions of the heuristic switching model and to estimate the IoC parameter from aggregate decisions of the experimental subjects. In particular, we construct a decision environment which is a stylized version of the framework laid 
down in the seminal work by Brock and Hommes (1997, 1998). This framework has served as a benchmark heuristic switching model in the literature ${ }^{1}$ but thus far its basic assumptions and implications have never been tested in a controlled laboratory experiment.

In our experiment we let subjects choose between a costly stabilizing heuristic and a cheap destabilizing heuristic. Payoffs associated with the choices depend upon the distribution of subjects over the heuristics. An important prediction of the heuristic switching model is that if costs for the stabilizing heuristic increase (relative to the costs for the destabilizing heuristic), the dynamics of the state variable (e.g., the price) and the distribution of agents over heuristics become unstable and complicated endogenous fluctuations may emerge. In our experiment we indeed find that an increase in costs for the stabilizing heuristic initially leads to the type of bubbles and crashes that are typical for the standard heuristic switching model. However, for the case of high costs we also find that, over time, the subjects adapt their behavior such that the dynamics becomes more stable and is consistent with a steady state of the model (although the dynamics are still much more volatile than in the low cost case). In particular, the estimated values of the intensity of choice parameter are much smaller for high costs than for low costs, which suggests that the characteristics of the economic environment are an important determinant of the value of this parameter. This finding is important because in the standard heuristic switching model the intensity of choice parameter is assumed to be exogenously given.

Our paper contributes to a growing empirical literature that tries to estimate the heuristic switching model from market or survey data. Goldbaum and Mizrach (2008), for example, estimate the IoC parameter on mutual fund allocations decisions, whereas Branch (2004) uses survey data to estimate a discrete choice model with switching between three heuristics. Boswijk, Hommes, and Manzan (2007) and Chiarella, He, and Zwinkels (2014) use U.S. stock price data to estimate a heuristic switching model with a fundamentalist heuristic and a trend-following or

\footnotetext{
${ }^{1}$ According to Google Scholar Brock and Hommes (1997) and Brock and Hommes (1998) together have been cited more than 3100 times as of December 5, 2017. Examples of theoretical contributions that build upon their framework can be found in Gaunersdorfer (2000), Chiarella, Dieci, and Gardini (2001), Brock, Hommes, and Wagener (2005), Branch and Evans (2006), Chiarella, Dieci, and Gardini (2006) and Panchenko, Gerasymchuk, and Pavlov (2013), among many others. A number of papers use the framework to study the effect of various financial market policies, such as the imposition of Tobin transaction taxes (Westerhoff and Dieci, 2006), increasing the number of financial derivatives (Brock, Hommes, and Wagener, 2009) and restricting short selling (Anufriev and Tuinstra, 2013).
} 
a chartist heuristic, respectively. Survey data of expectations in foreign exchange markets are used in Goldbaum and Zwinkels (2014). Finally, Cornea-Madeira, Hommes, and Massaro (2017) estimate a discrete choice model with switching between fundamentalists and random walk believers, using U.S. macroeconomic data. Although these contributions provide compelling evidence that the heuristic switching model performs well as a description of market behavior for different types of data, the drawback is that the IoC parameter in these models typically needs to be jointly estimated with other behavioral parameters (e.g., the heuristics are often parametrized as well). Several studies, including Boswijk, Hommes, and Manzan (2007), find that the estimated value of the IoC parameter is not significant. Studies that produce significant estimates of the IoC report very different values, depending on the specification of the heuristics. ${ }^{2}$ Finally, there is a large variation in the estimated value of the IoC parameter between studies. ${ }^{3}$ This uncertainty regarding the relevant range of values for this parameter, which plays such a crucial role in the dynamics of heuristic switching models, may deter policy-makers from using those models.

Laboratory experiments have the advantage that the experimenters can control both the market environment in which the subjects operate as well as the information these subjects have about that market environment. Therefore laboratory experiments can be used to obtain relatively clean data concerning the relationships under study. So-called 'Learning to Forecast' experiments (see, e.g., Hommes, Sonnemans, Tuinstra, and van de Velden, 2005, Hommes, Sonnemans, Tuinstra, and van de Velden, 2007 and Heemeijer, Hommes, Sonnemans, and Tuinstra, 2009, and Hommes, 2011 for an overview) have been used to estimate different forecasting heuristics that subjects typically use. An important feature of these Learning to Forecast experiments is that they take into account the self-referential characteristics of dynamic market environments. That is, expectations about the future value of an economic variable feed back into the actual realization of that variable,

\footnotetext{
${ }^{2}$ For instance, Goldbaum and Mizrach (2008) use the risk adjusted payoffs of investors and estimate the IoC parameter as $0.9,1.9$, and 6.53 for three different values of the risk aversion parameter. Cornea-Madeira, Hommes, and Massaro (2017) report values of the IoC parameter ranging from 1.99 to 9.26 , depending on the forecasting rules used by fundamentalists.

${ }^{3}$ Because the IoC parameter is not a scale-free parameter the variation in the estimated values of this parameter may be partly due to the differences in (average) performance levels. This conjecture is, however, difficult to verify in empirical studies as performance levels of the different heuristics are rarely reported. Recently, ter Ellen, Hommes, and Zwinkels (2017) proposed a model where switching is based on relative, instead of absolute, profit differences. This opens up the possibility to compare the estimated IoC for various asset classes, such as metals, real estate prices, and foreign exchange markets, directly. The estimates of the IoC in ter Ellen, Hommes, and Zwinkels (2017) still differ across markets, however.
} 
and thereby in part determine the behavior of that variable. This is in contrast to earlier experiments on expectation formation where participants have to predict an exogenously generated time series (see e.g., Schmalensee, 1976, Hey, 1994 and Kelley and Friedman, 2002).

Experiments can also be used to better understand how people exactly switch between different heuristics. A first step in that direction, which can be seen as complementary to the Learning to Forecast experiments where the heuristics themselves are estimated, is taken in the experiment conducted in Anufriev, Bao, and Tuinstra (2016). In that experiment subjects have to choose between different alternatives, where the payoffs associated with the alternatives are exogenously generated. The aggregate choice data are used to estimate different versions of the discrete choice model. The experiment presented in the current paper differs from the one in Anufriev, Bao, and Tuinstra (2016) in that payoffs are endogenously generated in this new experiment, and determined by the aggregate choices of the subjects. This setting therefore represents the typical heuristic switching model better.

The remainder of the paper is organized as follows. We start by introducing a stylized version of the heuristic switching model in Section 2. This model is a simplified version of the original Brock-Hommes model and we use it as a basis for our experimental environment. In Section 3 we discuss the experimental design and in Section 4 we formulate the hypotheses to be tested. In Section 5 we present the experimental results. We fit a simple discrete choice model to subjects' aggregate choices and argue that the IoC parameter changes over time in response to the decision environment. In addition, we show that our results are robust to increasing the number of subjects in the experimental groups and to increasing the number of decision periods. Concluding remarks are given in Section 6 .

\section{$2 \quad$ A stylized heuristic switching model}

This section introduces the stylized heuristic switching model that will be used in the laboratory experiment presented in this paper. Some more background on the heuristic switching model and a description of the main mechanism that leads to complicated dynamics in that model are given in Section 2.1. We subsequently describe the stylized heuristic switching model that we use in this paper and 
which consists of two parts: the dynamics of the state variable, which we discuss in Section 2.2, and the discrete choice model that describes switching between heuristics, which we discuss in Section 2.3.

\subsection{Complex dynamics from the interaction of heuristics}

The heuristic switching model was introduced in Brock and Hommes (1997). They consider a cobweb market where a large number of producers have to decide how much to supply of a (non-storable) commodity that takes one period to produce. The realized market price for the commodity will be the price for which consumer demand equals the aggregate supply of the producers, where the latter is determined by the individual price expectations of the producers. In Brock and Hommes (1997) it is assumed that there are two forecasting heuristics available to the suppliers: either naive expectations - where a producer uses the last observed price as his prediction for the next price, or rational expectations - where a producer knows the underlying market equilibrium condition, as well as the distribution of producers over the different heuristics, and uses this information to compute the market clearing price in the current period. Whereas the naive expectations heuristic uses very little (and publicly available) information, applying the rational expectations heuristic is more demanding. It requires substantially more information as well as the cognitive effort to process this information correctly. It therefore seems reasonable to assume that using the rational expectations heuristic comes at strictly higher (information) costs than using the naive expectations heuristic.

Every period producers decide which forecasting heuristic to use on the basis of relative past performance (after information costs have been deducted). It turns out that complicated dynamics may emerge if the sensitivity of producers with respect to the profit difference (i.e., producers' intensity of choice) of the heuristics is sufficiently high. The mechanism underlying these complicated dynamics can be described as follows. When the price is close to its steady state level, the forecasting heuristics give comparable predictions and, given their relative cost, producers have the incentive to choose the naive expectations heuristic. However, when almost all producers use naive expectations, the cobweb dynamics are unsta-

ble and market prices start to oscillate (see, e.g., Ezekiel, 1938). As a consequence, forecasting errors under naive expectations increase and producers tend to switch 
to the more profitable rational expectations heuristic. When enough producers use the rational expectations heuristic, prices converge to their steady state value and forecasting errors of both heuristics are similar again. As the rational expectations heuristic still comes with a cost, producers switch back to the naive expectations heuristic and the whole cycle repeats.

This type of mechanism, inducing complicated dynamics, works in many other market environments as well. Competition between fundamentalists and chartists in financial markets provides the most celebrated application, see, e.g., Brock and Hommes (1998), Chiarella and He (2003) and Chiarella, He, and Hommes (2006) for early examples and Hommes (2013) for an overview. It is important to note that the mechanism is qualitatively robust to changes in the set of heuristics, to the type of market institution (e.g., Walrasian equilibrium clearing, a market maker that adjusts prices, or continuous double auctions) and to the direction of the expectations feedback. ${ }^{4}$ Generally, if the IoC parameter and/or the costs for the stabilizing heuristic are high enough, the fraction of agents using the stabilizing heuristic at the steady state is going to be low and will not be sufficient to stabilize the dynamics. The steady state will then be unstable and prices as well as the distribution of traders over the heuristics will fluctuate endogenously. The precise characteristics of the dynamics as well as the threshold values of the IoC parameter and the costs for the stabilizing heuristic will depend on the features of the underlying market environment.

\subsection{Dynamics of the state variable and performance of heuristics}

Our aim is to test the key assumptions and implications of the heuristic switching model described above in a laboratory experiment with paid human subjects. Laboratory experiments have the advantage that they generate clean data on choice behavior, that information given to the subjects, as well as the underlying model, are under control of the experimenters, and that subjects have well-defined monetary incentives. In addition, laboratory experiments can be replicated. At

\footnotetext{
${ }^{4}$ See Gaunersdorfer, Hommes, and Wagener (2008), Anufriev and Panchenko (2009), and Hommes (2005), respectively. Positive expectations feedback, where actual prices respond positively to an increase in the average expected price, is typical for financial markets, whereas negative expectations feedback, with a negative price response to an increase in price expectations, is common for supply-side driven markets, such as the cobweb market discussed above.
} 
the same time, it is important to use decision environments that are not too complicated, in order to ensure that subjects have a good understanding of the task they are asked to perform. For this reason we want to use a stylized heuristic switching model that still exhibits the main mechanism of the models described above, but which is straightforward to implement in a laboratory experiment.

To that end, consider an economy where agents repeatedly choose between two profitable alternatives ('heuristics'), $A$ and $B$. The profits generated by these alternatives are determined by an underlying state variable $x_{t}$. More specifically, profits of choosing $A$ and $B$ in period $t$ are given by

$$
\pi_{A, t}=W_{A}+\gamma_{A} x_{t}^{2}, \quad \text { and } \quad \pi_{B, t}=W_{B}-\gamma_{B} x_{t}^{2},
$$

respectively, where $W_{A}, W_{B}, \gamma_{A}$ and $\gamma_{B}$ are nonnegative parameters, with $\gamma_{A}+$ $\gamma_{B}=1$. Note that profits for alternative $A$ depend positively on the deviation of the state variable from zero, and the other way around for alternative $B .^{5}$

The evolution of the state variable $x_{t}$ depends upon the distribution of agents over the alternatives $A$ and $B$. In particular, we consider

$$
x_{t}=\lambda n_{B, t} x_{t-1}+\epsilon_{t},
$$

where $n_{B, t} \in[0,1]$ is the fraction of the population of agents that chooses alternative $B$ in period $t, \lambda$ is the feedback coefficient and $\epsilon_{t}$ is a small idiosyncratic random shock. The sign of $\lambda$ determines whether the feedback in the market is negative or positive, with $\lambda<0$ representing the cobweb model of Brock and Hommes (1997) and $\lambda>0$ representing the asset pricing model of Brock and Hommes (1998). In both cases, the state variable $x$ can be interpreted as the deviation of the price from its fundamental value.

The mechanism leading to endogenous fluctuations, discussed in Section 2.1 for the case of the cobweb model, but also relevant for financial markets, is preserved in the system consisting of equations (1) and (2), provided that $W_{B}>W_{A}$ and

\footnotetext{
${ }^{5}$ In the standard model only the difference between profits matters for agents, cf. Eq. (4) in Section 2.3. In our setup this difference is $\pi_{A, t}-\pi_{B, t}=W_{A}-W_{B}+x_{t}^{2}$, which, due to $\gamma_{A}+\gamma_{B}=1$, does not depend on the exact values of $\gamma_{A}$ and $\gamma_{B}$. For the experiment we choose the values of $W_{B}$ and $\gamma_{B}$ such that profits of alternative $B$ remain positive for a relatively large range of values of $x_{t}$. This is done in order to minimize the number of periods in the experiment where payoffs for alternative $B$ become negative. If in some period $t$ the state variable $x_{t}$ is so large that $\pi_{B, t}<0$, we assign payoff 0 to alternative $B$ for that period. This approach, which ensures that participating subjects have positive earnings, is common for laboratory experiments.
} 
$|\lambda|>1$. To see this, note that when the state variable $x_{t}$ equals its steady state value of $x^{*}=0$, alternative $B$ is more profitable than alternative $A$ (at this point $\left.\pi_{B}=W_{B}>W_{A}=\pi_{A}\right)$ and therefore attracts agents. Thus for low absolute values of the state variable, fraction $n_{B, t}$ will increase over time. However, when $n_{B, t}$ becomes large enough (in particular, when $\left|\lambda n_{B, t}\right|>1$ ), the dynamics of the state variable become unstable and $x_{t}$ will diverge away from its steady state value. When the deviation of $x_{t}$ from zero becomes sufficiently large (to be specific, when $\left.\left|x_{t}\right|>\sqrt{W_{B}-W_{A}}\right)$ alternative $A$ becomes more profitable and agents will tend to switch to that alternative again, which stabilizes the dynamics, and so on.

The model presented here, therefore, corresponds to a stylized version of the heuristic switching model described above, with alternative $A$ playing the role of the costly stabilizing heuristic and alternative $B$ playing the role of the cheap destabilizing heuristic. In addition, we can think of $W_{B}-W_{A}$ as the costs for heuristic $A$, because it corresponds to the difference in profits between the cheap heuristic $B$ and the costly heuristic $A$, when $x_{t}=0$, that is, at the steady state. In the remainder of this paper we will therefore denote costs for using heuristic $A$ (relative to the costs for using heuristic $B$ ) by $C=W_{B}-W_{A}$, which we will assume to be strictly positive. ${ }^{6}$

\subsection{The discrete choice model}

The model given by equations (1)-(2) still lacks a description of how agents choose between alternatives $A$ and $B$ when they know the past performance of alternatives (i.e., profits $\pi_{A, t}$ and $\pi_{B, t}$ ) but not the underlying profit generating mechanism. The experiment presented in this paper is designed specifically to investigate that decision, using human subjects. The most common approach to this choice problem in the literature on heuristic switching models is the so-called discrete choice model, which - in one of its most basic forms - looks as follows. Let $P_{A, t}$ and $P_{B, t}$ be the probabilities that an agent chooses alternative $A$ and $B$ in period $t$,

\footnotetext{
${ }^{6}$ It is worthwhile to stress one difference with the models from Brock and Hommes (1997, 1998). In our stylized version the value of the state variable in period $t$ depends upon $n_{B, t}$. This allows us to write the model as a simpler one-dimensional dynamical system, see Eq. (5), as opposed to the two-dimensional dynamical systems in Brock and Hommes (1997, 1998). The dynamics, as well as the mechanism driving them, is qualitatively equivalent between the two settings. See Appendix A for a formal analysis.
} 
respectively. Probability $P_{B, t}$ is specified as

$$
P_{B, t}=\frac{\exp \left[\beta \pi_{B, t-1}\right]}{\exp \left[\alpha+\beta \pi_{A, t-1}\right]+\exp \left[\beta \pi_{B, t-1}\right]}=\frac{1}{1+\exp \left[\alpha+\beta\left(\pi_{A, t-1}-\pi_{B, t-1}\right)\right]},
$$

with $P_{A, t}=1-P_{B, t}$. Here $\beta \geq 0$ is the Intensity of Choice (IoC) parameter and $\alpha$ is a parameter that measures the so-called predisposition effect. The intuition behind the discrete choice model is that an increase in the performance of one alternative, relative to the other alternative, increases the probability that an individual agent chooses the former. The IoC parameter $\beta$ measures how sensitive this probability is with respect to the performance difference. The predisposition effect measures a possible bias that agents have towards one of the alternatives: a positive value of $\alpha$ implies that alternative $A$ is chosen with a higher probability than alternative $B$ when their performance is the same. Similarly, a negative value of $\alpha$ implies that agents are biased towards alternative $B$.

Assuming that there are many agents, with all of them choosing between alternatives $A$ and $B$ according to (3), the Law of Large Numbers implies that the fraction of agents choosing alternative $B$ is given by

$$
n_{B, t}=\frac{1}{1+\exp \left[\alpha+\beta\left(\pi_{A, t-1}-\pi_{B, t-1}\right)\right]},
$$

with $n_{A, t}=1-n_{B, t}$.

The canonical discrete choice model, as used in Brock and Hommes (1997, 1998) and in the vast majority of subsequent contributions, is given by (4) with $\alpha=0$. However, estimated discrete choice models on survey data (Branch, 2004) and experimental data (Anufriev, Bao, and Tuinstra, 2016) suggest that the predisposition effect plays an important role in explaining human behavior and therefore we add it here as well. ${ }^{7}$

We can now complete the model presented in Section 2.2 by assuming that (4) describes how the population of agents chooses between alternatives. Substituting

\footnotetext{
${ }^{7}$ There exist alternative formulations of the discrete choice model, for example where performance is measured by a weighted average of past profits (instead of only the profits from the previous period) or where updating is asynchronous (that is, agents do not have the opportunity to change between alternatives every period), see Anufriev and Hommes (2012a). As an alternative to the discrete choice model, the evolution of the fraction choosing one particular alternative can be modelled by the (exponential) replicator dynamics, see, e.g., Dindo and Tuinstra (2011), Tuinstra, Wegener, and Westerhoff (2014) and Schmitt, Tuinstra, and Westerhoff (2017). Derived from a process of imitation, this evolutionary model leads to a more sluggish adaptation of fractions, which is similar to the effect of asynchronous updating.
} 
(1) and (4) in (2) and using $\gamma_{A}+\gamma_{B}=1$ and $C=W_{B}-W_{A}$, we find that the state variable $x_{t}$ evolves according to

$$
x_{t}=f\left(x_{t-1}\right)+\epsilon_{t}=\frac{\lambda x_{t-1}}{1+\exp \left[\alpha+\beta\left(x_{t-1}^{2}-C\right)\right]}+\epsilon_{t} .
$$

Appendix A analyzes the dynamics of this non-linear model and shows that the key properties of the heuristic switching framework outlined in Brock and Hommes (1997, 1998) are preserved. It turns out that the dynamics of the state variable, given by the first order difference equation (5), may give rise to complicated dynamics, even in absence of random shocks (i.e., setting $\epsilon_{t}=0$ ). This depends in particular upon the structural parameters $\lambda$ and $C$ and the behavioral parameters $\alpha$ and $\beta$.

Note that when the dynamics of the state variable are given by (5), the fraction of choices for alternative $B$ evolves as

$$
n_{B, t}=\frac{1}{1+\exp \left[\alpha+\beta\left(x_{t-1}^{2}-C\right)\right]} .
$$

When the state variable equals $x^{*}=0$, the fraction of $B$-choices is given by $n_{B}^{*}=[1+\exp (\alpha-\beta C)]^{-1}$. The following finding (which focuses on the case $\lambda>0$ ) follows from the more general result proven in Appendix A.

Proposition 2.1. Consider the first order difference equation (5) with $\epsilon_{t}=0$. For $0<\lambda<1$ there is a unique globally stable zero steady state, $x^{*}=0$. For $\lambda>1$ the zero steady state is unique and locally stable as long as $\lambda n_{B}^{*}<1$ and unstable when $\lambda n_{B}^{*}>1$. Moreover, when $\lambda n_{B}^{*}>1$, two non-zero steady states $x^{+}$ and $x^{-}$exist, with $x^{+}=\sqrt{C+(\ln (\lambda-1)-\alpha) / \beta}$ and $x^{-}=-x^{+}$. The associated steady state fractions are $n_{B}^{+}=n_{B}^{-}=\lambda^{-1}$. The non-zero steady states are locally stable if $\beta\left(1-n_{B}^{+}\right)\left(x^{+}\right)^{2}<1$.

Clearly, an increase in the fraction of agents using alternative $B$ inhibits stability of the zero steady state (recall that alternative $B$ corresponds to the cheap but destabilizing heuristic). Also note that we assume that $C=W_{B}-W_{A}$, the costs for using the more sophisticated and stabilizing rule $A$, is positive. Clearly, an increase in $C$ increases $n_{B}^{*}$ and destabilizes the zero steady state, because - at the steady state - more agents use the destabilizing rule. Likewise, an increase in the IoC parameter $\beta$ will destabilize the dynamics, since this also increases the fraction of agents using alternative $B$ (since they will do better at the steady 

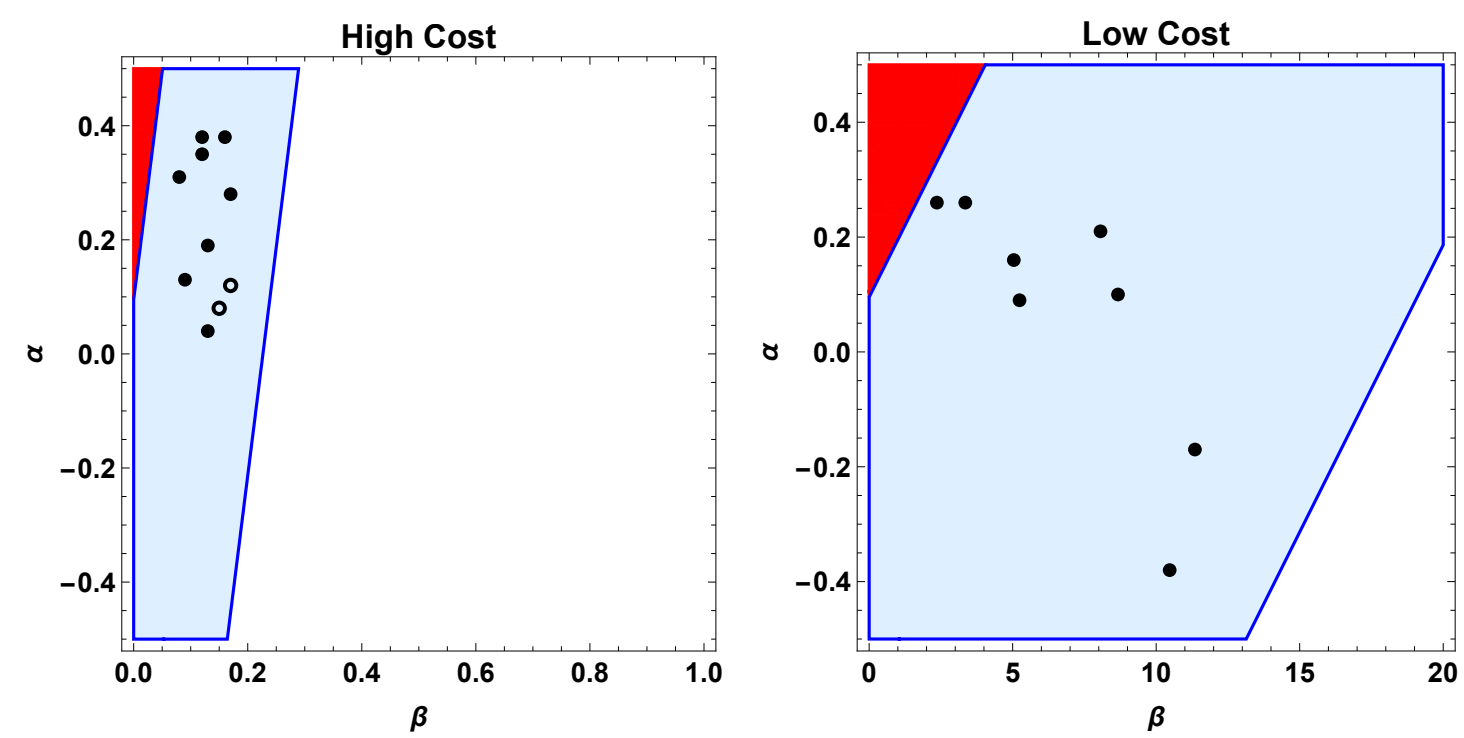

Figure 1: Bifurcation diagrams in $(\beta, \alpha)$-coordinates for the stylized heuristic switching model, showing the regions of stability for the zero steady state (dark filled region), and non-zero steady states (light filled region). Left: Parameters corresponding to the High blocks in the experiment. Right: Parameters corresponding to the Low blocks in the experiment. Black points show the values of $(\beta, \alpha)$ estimated on subjects' choice data (see Section 5.2). The two black disks on the left panel show the estimated values from the High Long treatment (see Section 5.3).

state). An increase in $\alpha$, on the other hand, will promote stability. If the zero steady state loses stability, for example because $\beta$ increases, this occurs through a so-called pitchfork bifurcation. In this bifurcation two new, non-zero, steady states are created that are locally stable. The absolute deviation of these two steady states from zero is the same and depends upon the behavioral parameters of the model. With a further increase of $\beta$ or $C$, these non-zero steady states become unstable as well. ${ }^{8}$

Fig. 1 shows the stability regions of the different steady states for the case of high costs, $C=8$ (left panel) and low costs, $C=0.1$ (right panel). The value of the parameter $\lambda$ is 2.1, which is the value that will be used in the experiment. Note that for high costs the stability regions (both of the zero and of the non-zero steady states) are much smaller than for low costs (also note that the scale on the horizontal axis is very different).

The aim of the experiment in this paper, outlined below, is to verify that

\footnotetext{
${ }^{8}$ Note that for the case of $\lambda<0$ we get a similar stability condition. However, in this case, instead of two non-zero steady states, a period two cycle $\left\{x^{-}, x^{+}\right\}$, with $x^{-}=f\left(x^{+}\right)$and $x^{+}=f\left(x^{-}\right)$, is created when the zero steady state $x^{*}$ loses stability, see Appendix A.
} 
the aggregate dynamics in our experiment will reproduce the patterns that are predicted by the heuristic switching model. In addition, we want to fit the discrete choice model on the aggregate choice data, and in particular estimate the relevant values of the intensity of choice parameter $\beta$ and the predisposition parameter $\alpha$.

\section{Experimental Design}

The experiment took place in June 2016 and October 2017 at the University of Technology Sydney Behavioral Laboratory. In total 80 subjects were recruited for four sessions, with 20 subjects participating in each session. ${ }^{9}$ Most subjects are undergraduate students in economics and finance, and no subject participated in more than one session. Each session lasted approximately 90 minutes and subjects earned 25 Australian dollars (AUD) on average.

The task of the subjects in the experiment is to make a choice between two investment alternatives, labeled $A$ and $B$. In the beginning of each session the 20 subjects that participate in the session are randomly divided in two groups of 10 . Then, for 40 consecutive periods subjects make their binary choices, with their payoffs dependent on the distribution of choices of the 10 subjects in their group. After the first block of 40 decision periods is finished, subjects in the session are randomly rematched to form two new fixed groups of 10 subjects. During the second block, the subjects have to make their binary decisions for another 40 periods, with their payofs depending on the choices of the subjects in their new group of 10 subjects.

At the beginning of every period subjects are provided with information about the past profits of the two alternatives, both in the form of a table and a graph, see Fig. 2. In addition they are informed about their own previous choices. As soon as all subjects in the group have made their decision for period $t$, the actual profits for the two alternatives are generated by (1), where the value $x_{t}$ of the state variable ${ }^{10}$ is determined by (2). The profits of both alternatives are then

\footnotetext{
${ }^{9}$ The Online Recruitment System for Economic Experiments (ORSEE), see Greiner (2015), was used for recruiting participants. The experiment was conducted using the z-Tree software, see Fischbacher (2007). Two sessions of an additional treatment, with more periods and larger groups, took place in September 2017. We will discuss the results for this additional treatment in Section 5.3.

${ }^{10}$ As explained in Section 2.2, this state variable can be thought of as the deviation of the price from the fundamental value. We do not ask subjects to predict the values of this variable
} 


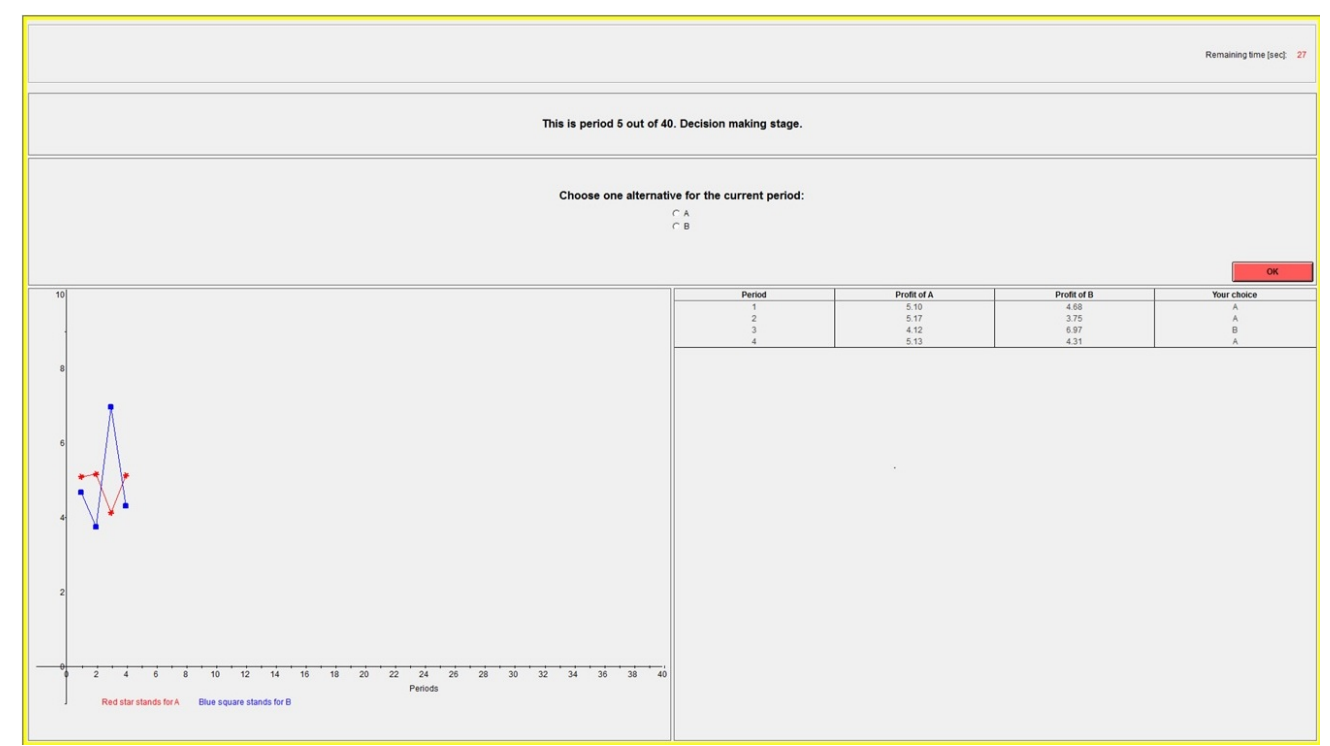

Figure 2: An example of the computer screen with interface elements. The upper part of the screen is used to submit a decision by clicking on one of two radio buttons, $A$ or $B$. The lower part represents the history of profits for alternatives $A$ and $B$ in two formats: a graph on the left and a table on the right. The last column in the table shows the past choices of the subject.

shown to the subjects together with their choice in period $t$. Subsequently they are asked to make their choice for period $t+1$.

For both blocks in each session we choose $\lambda=2.1$ (implying that the dynamics are unstable if, at equilibrium, at least half of the participants chooses alternative $B$, see Proposition 2.1), $\gamma_{A}=0.6$ and $\gamma_{B}=0.4$. The random shocks are IID distributed according to $\epsilon_{t} \sim N(0,0.02)$. We use the same realization of random shocks for each block, and for each session. The only difference between blocks are the values of $W_{A}$ and $W_{B}$, that are chosen in order to generate blocks with different costs, $C=W_{B}-W_{A}$, for the stabilizing heuristic. For the High blocks we choose $W_{A}=1$ and $W_{B}=9$ (i.e., high cost is given by $C=8$ ) and for the Low blocks we choose $W_{A}=4.95$ and $W_{B}=5.05$ (i.e., low cost is given by $C=0.1$ ).

The two treatments in our experiment, with two sessions each, only differ in the order of the blocks: treatment High-Low starts with a block with $C=8$, followed by a block with $C=0.1$, whereas the order is reversed for treatment Low-High. ${ }^{11}$ We therefore have eight groups that make decisions in a High block

(as they would do in the Learning to Forecast experiments). In fact, we do not even show the evolution of this variable to the subjects. This design has been chosen to focus exclusively on testing the discrete choice model, which assumes subjects only respond to the performance of the heuristics.

${ }^{11}$ Treatment High-Low was implemented in sessions 1 and 3, treatment Low-High was imple- 
and eight groups that make decisions in a Low block. Each of these sixteen groups consists of 10 subjects, with each subject participating in exactly one High and one Low block. In the remainder we will identify groups by block, session and group number (e.g., "High: session 1, group 2" refers to the second group in the first block of the first session).

By the end of the experiment, subjects are paid for their decisions according to the following procedure. For every subject we randomly choose two periods from the first block and two periods from the second block. The sum of the payoffs corresponding to the decision of the subject in these four periods is divided by two. This constitutes the subject's earnings in Australian dollars. In addition, each subject receives a 10 AUD participation fee. The procedure ensures that subjects have the financial incentive to make the best possible decision in every period of the experiment. Experimental instructions are provided in Appendix B. ${ }^{12}$

\section{Hypotheses}

If the discrete choice model (4) gives a good description of the aggregate behavior of subjects, then (5) and (6) should provide a good approximation ${ }^{13}$ of the dynamics of the fraction $n_{B, t}$ and the state variable $x_{t}$. The dynamics will depend upon the specific characteristics of the economic decision problem $\left(\lambda, \gamma_{A}, \gamma_{B}, W_{A}\right.$ and $W_{B}$ ), which are chosen by the experimenters, and upon the behavioral parameters $\alpha$ and $\beta$, which describe the subjects' decision making. Since $C=W_{B}-W_{A}>0$ in both blocks, in equilibrium we will have $n_{B}^{*}>1 / 2$ (assuming $\alpha$ is not very high and $\beta>0)$. Given that $\lambda=2.1$, we therefore expect the zero steady state, $\left(x^{*}, n_{B}^{*}\right)=\left(0,[1+\exp (\alpha-\beta C)]^{-1}\right)$, to be locally unstable in both the High and the Low blocks, see Proposition 2.1. Moreover, the values of $W_{A}$ and $W_{B}$ in the two blocks are such that for a large range of values of $\alpha$ and $\beta$ the steady states $\left(x^{+}, n^{+}\right)$and $\left(x^{-}, n^{-}\right)$will be stable for the Low blocks, but unstable for the High

\footnotetext{
mented in sessions 2 and 4.

${ }^{12}$ Before the experiment starts subjects are required to solve a short quiz which is designed to check their understanding of the feedback effects and remuneration procedure. After the experiment, subjects are given a questionnaire that focuses on background information, such as demographic characteristics and field of study. The quiz and the questionnaire are available from the authors upon request.

${ }^{13}$ In the model fraction $n_{B, t}$ can take on any real value in the interval $[0,1]$. In the experiment, however, there are 10 subjects in a group, and, therefore, this fraction can only take on 11 values $(0,0.1,0.2, \ldots, 1)$. Hence, $(5)$ and $(6)$ can only provide an approximation to the model dynamics.
} 

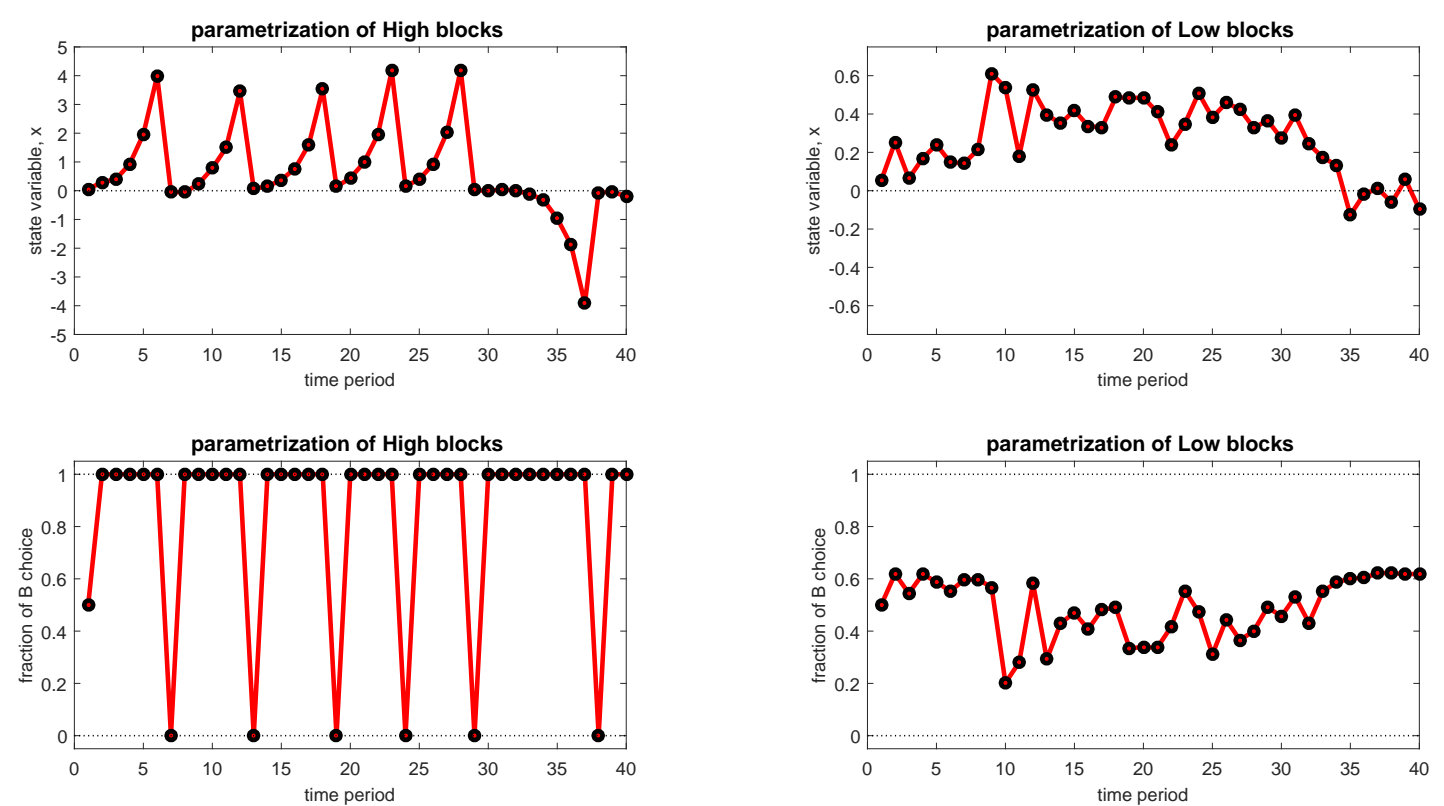

Figure 3: Simulations of the stylized heuristic switching model with $\lambda=2.1$. Left: High blocks parametrization, $C=8$. Right: Low blocks parametrization, $C=0.1$. Upper panels: Dynamics of state variable, $x$. Lower panels: Dynamics of fractions of $B$-choices, $n_{B}$.

blocks, see Fig. 1.

Fig. 3 illustrates the simulated dynamics of the stylized heuristic switching model (5)-(6) for the values of the structural parameters that we use in the experiment, and with the same realization of the random shocks $\epsilon_{t}$ that is used in the experiment. For these simulations we set the behavioral parameters to $\alpha=0$ and $\beta=5$. The left panels show the dynamics of $x$ and $n_{B}$ in the High blocks. We observe a pattern familiar from the original model of Brock and Hommes (1998) with endogenous bubbles and crashes of the state variable (corresponding to the deviation of the price from the fundamental asset value in their framework). Note that due to the small random shocks, the realized state variable may become negative when it approaches zero. The right panels show the same dynamics for the Low blocks. Due to positive values of $\epsilon$ in the first couple of periods, the state variable becomes positive and stays close to the steady state value $x^{+}=\sqrt{\ln (1.1) / 5+0.1} \approx 0.345$ thereafter, although it may occasionally 'escape' from that steady state when a sufficiently low value of the noise term $\epsilon$ is realized (which happens in period $t=35$ ).

The specific time series shown in Fig. 3 depend on the specific values of $\alpha$ and $\beta$, but the main properties of the model, i.e., instability of all steady states with 
endogenous bubbles and crashes for the High blocks and stability of the non-zero steady state for the Low blocks, hold for a large range of values of these behavioral parameters. Thus, if choice behavior is governed by the same heuristic switching model in both environments we expect less stability and more volatile dynamics in the High blocks than in the Low blocks, both in terms of the fraction of subjects choosing $B$, and in the state variable $x_{t}$. This leads to our first hypothesis on the dynamics of $n_{B, t}$ and $x_{t}$.

Hypothesis 1. There is a substantial difference in the volatility of both $n_{B, t}$ and $x_{t}$ between the High blocks and the Low blocks.

If Hypothesis 1 is not rejected, it implies that the qualitative predictions of the heuristic switching model are confirmed. The next step is to investigate whether the discrete choice model (4) also gives a good quantitative description of the data. To that end, we fit the discrete choice model on the aggregate data. This gives our next hypothesis.

Hypothesis 2. The endogenous variable $n_{B, t}$ can be described by a discrete choice model with one lag and a predisposition effect.

If this hypothesis is confirmed, it provides experimental evidence for the relevance of the discrete choice model (4) as a description of aggregate decision making, and would thereby lend support to the use of heuristic switching models. Note that in Anufriev, Bao, and Tuinstra (2016) it was established that a discrete choice model with one lag and a predisposition effect is relatively successful in describing experimental data in a setting where payoffs for the different alternatives are exogenously generated. Hypothesis 2 checks whether this is also the case when there is feedback from subjects' decisions to the payoffs.

Ideally, the estimated discrete choice model - if it provides a good description of the data - is similar for the High and the Low blocks. Comparable values of the model's behavioral parameters would suggest that human decision making is independent of the specifics of the economic environment and governed by the same underlying laws. Indeed, Hypothesis 1 implicitly assumes that the decision process is the same for the High and Low blocks and so the variation in the dynamics occurs because of the difference in the other characteristics of the High and Low blocks (i.e., the difference in costs $C=W_{B}-W_{A}$ ). Our third and final hypothesis deals with investigating this issue. 
Hypothesis 3. There is no significant difference between the discrete choice models estimated on data from the High blocks, and the discrete choice models estimated on data from the Low blocks.

In the next section we present the experimental data and test these three hypotheses.

\section{Experimental results on switching}

In this section we will discuss the experimental results. We start out with presenting the experimental data in Section 5.1. We will also provide some descriptive statistics and test Hypothesis 1 in that section. In Section 5.1 we estimate the discrete choice model on the experimental data and discuss Hypotheses 2 and 3. Finally, Section 5.3 is devoted to the analysis of an additional treatment with high costs, which features larger groups and more periods.

\subsection{The experimental data}

First, we depict the evolution over time of the fraction $n_{B, t}$ of subjects choosing alternative $B$. Figs. 4 and 5 show this fraction in the High and Low blocks, respectively. A quick visual inspection of these figures suggests that the time series of $n_{B, t}$ may indeed be slightly more volatile in the High blocks. For example, out of $8 \times 40$ observations the boundary values of 0 and 1 are reached 23 times $(7.2 \%)$ in the High blocks, whereas they are only reached 10 times (3.1\%) in the Low blocks. However, the standard deviations of $n_{B, t}$ in the different groups, reported in Table 1, appear to be roughly similar for the High and the Low blocks. Indeed, the difference in standard deviations between High and Low blocks is not statistically significant at the $5 \%$ level. $^{14}$

To further investigate possible differences in the fractions, the top panels of Fig. 6 show the histograms of the fraction $n_{B, t}$ of subjects choosing alternative $B$ in

\footnotetext{
${ }^{14}$ The Ansari-Bradley test, which is suitable for distributions with similar means and shapes, gives a $p$-value of 0.143 , implying that we cannot reject the hypothesis of equal standard deviations of the two distributions. Note that, in order to preserve independence of the observations, we only used the first block in each session for this test (i.e., the four High blocks from Sessions 1 and 3 and the four Low blocks from Sessions 2 and 4). For all other test statistics (unless noted otherwise) we pool the data from the first and second High (respectively Low) blocks.
} 

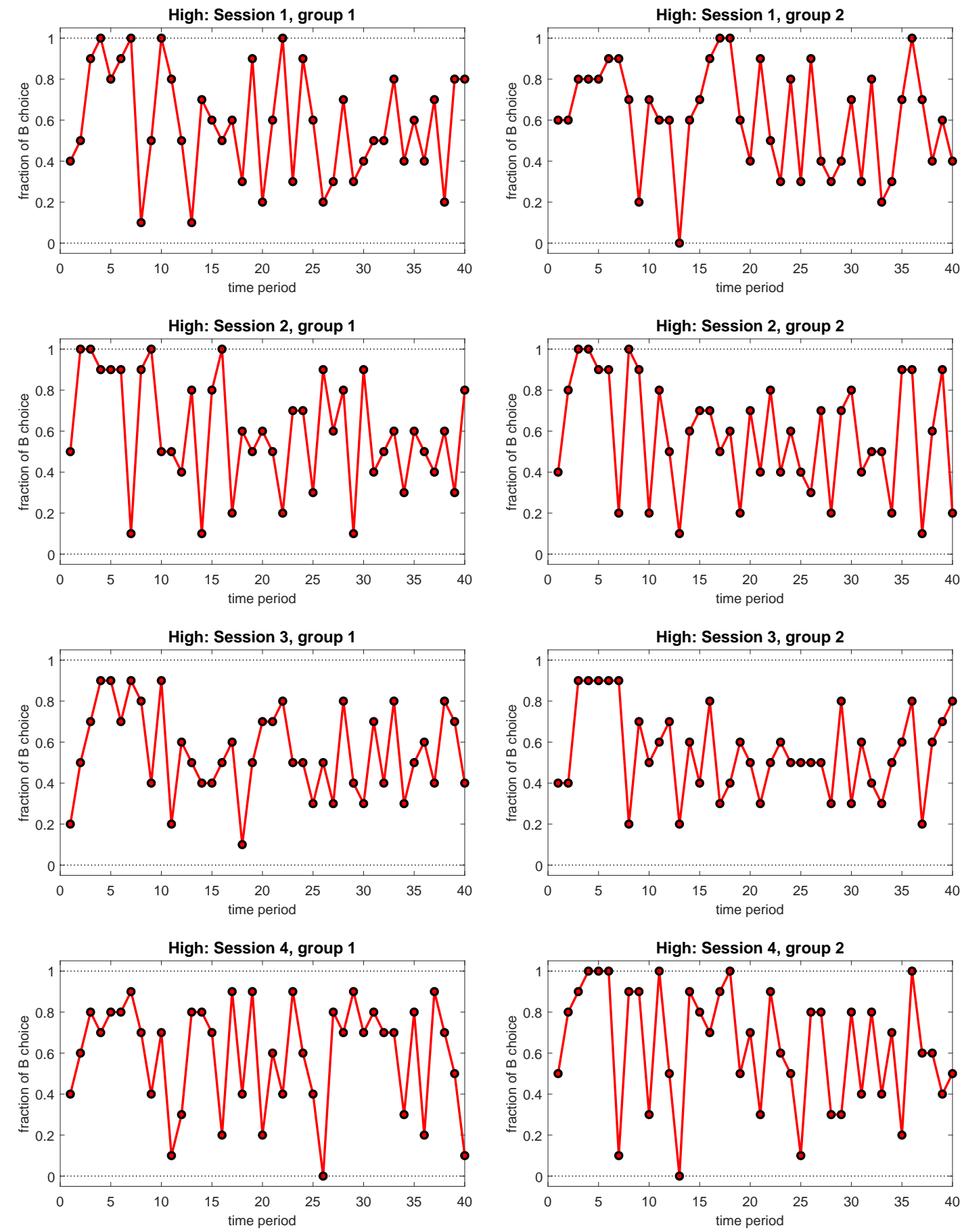

Figure 4: Fraction of choices of alternative $B$ in High blocks. 

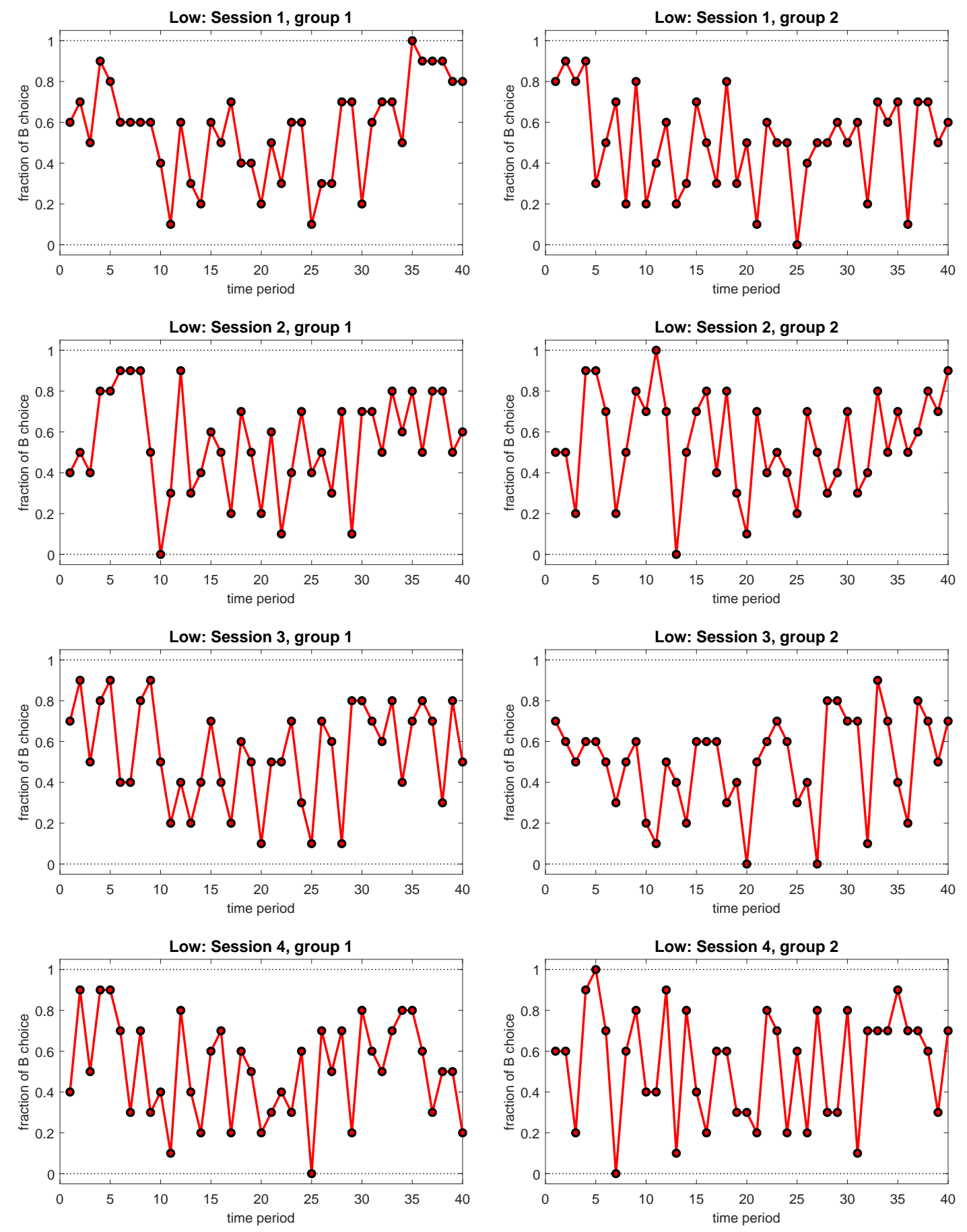

Figure 5: Fraction of choices of alternative $B$ in Low blocks. 


\begin{tabular}{|c|c|c|c|c|c|}
\hline & Data & \multicolumn{2}{|c|}{ Fraction of B-choices, $n_{B}$} & \multicolumn{2}{|c|}{ State variable, $x$} \\
\hline \multirow{9}{*}{ High } & Session 1. Group 1 & 0.58 & 0.27 & 2.43 & 1.52 \\
\hline & Session 1. Group 2 & 0.61 & 0.25 & 2.26 & 1.52 \\
\hline & Session 2. Group 1 & 0.60 & 0.27 & 2.51 & 1.47 \\
\hline & Session 2. Group 2 & 0.58 & 0.28 & 2.39 & 1.27 \\
\hline & Session 3. Group 1 & 0.55 & 0.22 & 2.33 & 1.27 \\
\hline & Session 3. Group 2 & 0.55 & 0.21 & 2.35 & 1.11 \\
\hline & Session 4. Group 1 & 0.60 & 0.26 & 2.02 & 1.45 \\
\hline & Session 4. Group 2 & 0.64 & 0.29 & 2.15 & 1.42 \\
\hline & All High groups & 0.59 & 0.26 & 2.43 & 1.52 \\
\hline \multirow{2}{*}{ High Long } & Session 5. Group 1 & 0.53 & 0.18 & 2.62 & 0.92 \\
\hline & Session 6. Group 1 & 0.55 & 0.21 & 2.48 & 1.06 \\
\hline \multirow{9}{*}{ Low } & Session 1. Group 1 & 0.56 & 0.23 & 0.26 & 0.22 \\
\hline & Session 1. Group 2 & 0.51 & 0.23 & 0.26 & 0.24 \\
\hline & Session 2. Group 1 & 0.55 & 0.24 & 0.17 & 0.21 \\
\hline & Session 2. Group 2 & 0.56 & 0.24 & 0.13 & 0.28 \\
\hline & Session 3. Group 1 & 0.55 & 0.24 & 0.32 & 0.22 \\
\hline & Session 3. Group 2 & 0.50 & 0.23 & 0.12 & 0.25 \\
\hline & Session 4. Group 1 & 0.51 & 0.24 & 0.31 & 0.18 \\
\hline & Session 4. Group 2 & 0.54 & 0.27 & 0.16 & 0.32 \\
\hline & All Low groups & 0.53 & 0.24 & 0.26 & 0.22 \\
\hline
\end{tabular}

Table 1: Descriptive statistics of the fraction $n_{B}$ and the state variable $x$.

the High and Low blocks, pooled over all eight groups. These histograms also show a small, but distinct, difference between the two types of blocks. The distribution of choices in the Low blocks has a clear peak around 0.5 - 0.7 (containing around half of the observations), whereas the distribution of fractions in the High blocks is much more evenly spread with substantially more observations of fractions $n_{B, t}$ close to 1 . In fact, the hypothesis of equality of the two distributions is rejected at the $5 \%$ level. $^{15}$

It is clear from the histograms of the fraction of $B$-choices that, although there is a difference between the High and Low blocks in our experiment, this difference is smaller than we would expect in case of stable and unstable dynamics in the heuristic switching model. Consider, for example, the time series of fractions given in the lower panels of Fig. 3 that are generated with $\alpha=0$ and $\beta=5$. The histogram of fractions corresponding to the lower left panel of Fig. 3 (High cost parametrization) will be bimodal, with many observations close to 0 or close to 1, whereas the histogram of fractions corresponding to the lower right panel (Low cost parametrization) will be single-peaked. Whereas the latter is indeed consistent with the histogram for our Low block groups in Fig. 6, the histogram

\footnotetext{
${ }^{15}$ The Kolmogorov-Smirnov test for equality of distributions gives a $p$-value of 0.012 .
} 

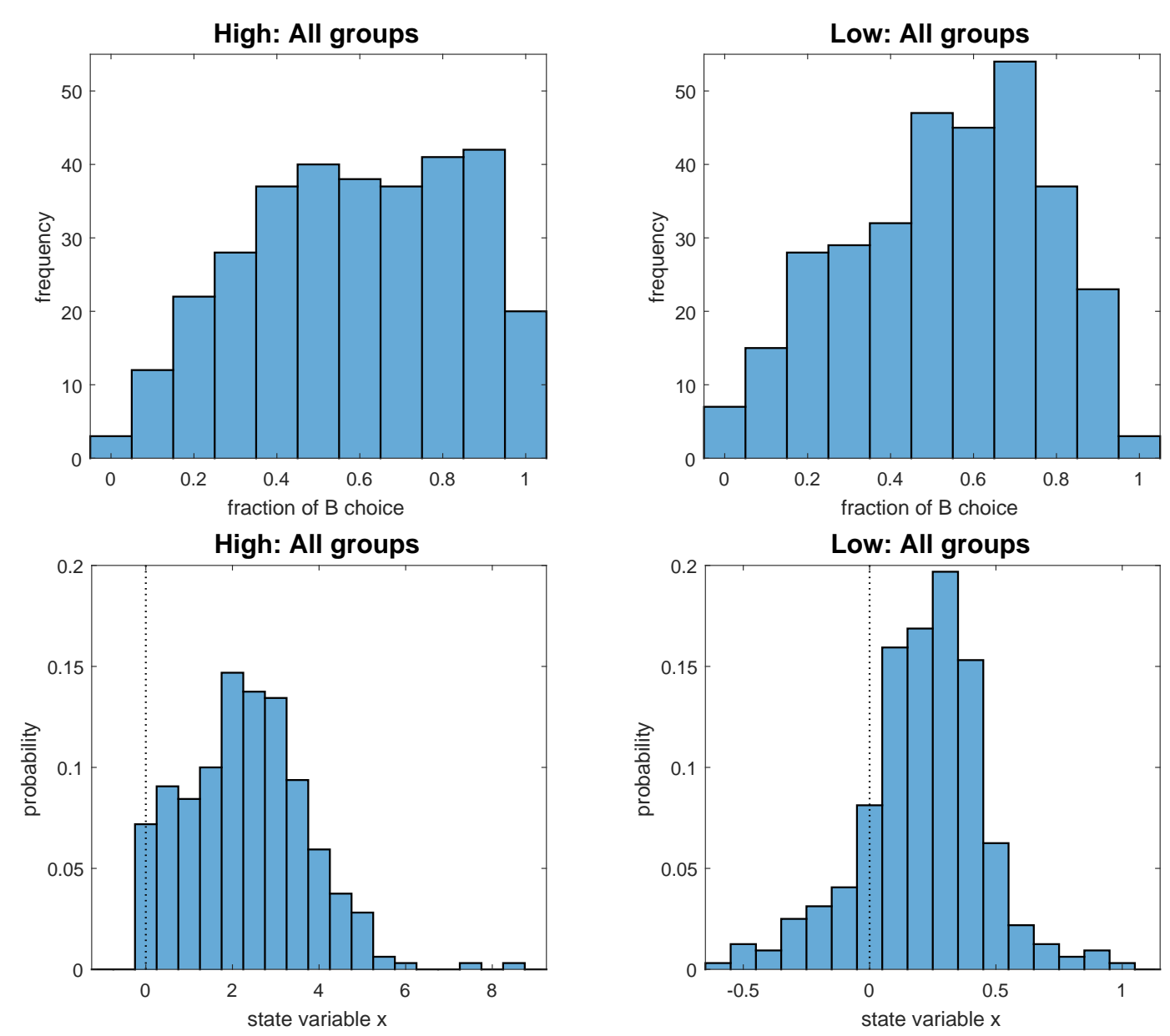

Figure 6: Histogram of fraction of $B$-choices (Upper panels). Density histogram of state variable, $x$ (Lower panels). Left: High blocks. Right: Low blocks. The vertical dashed lines in the lower panels indicate the zero steady state of $x$.

for the High block groups can hardly be described as bimodal.

Because the fraction of subjects choosing alternative $B$ is high in many periods, deviations of $x_{t}$ from zero should be quite persistent, at least in those periods, see Eq. (2). This is confirmed by inspection of Fig. 7 which shows the time series of $x_{t}$ in all blocks of each session. Clearly, the mean and variance of $x_{t}$ are much higher for the High blocks than for the Low blocks, as can also be seen from the last two columns of Table 1. Both the difference in means and the difference in standard deviations is statistically significant at the $5 \%$ level. ${ }^{16}$ Moreover, the mean values of $x_{t}$, both in the High and in the Low blocks, are significantly different from zero

\footnotetext{
${ }^{16}$ The Ansari-Bradley test of equal standard deviations (applied to the first blocks of each session again) gives a $p$-value of 0.003 . Since the data looks closer to a normal distribution now, see the lower panels of Fig. 6, we also use an $F$-test for equal variances, which returns a $p$-value of 0.000 . Similarly, the $p$-value of the $t$-test of equal means is 0.000 . Finally, the Kolmogorov-Smirnov test for equality of distributions gives a $p$-value of 0.000 .
} 

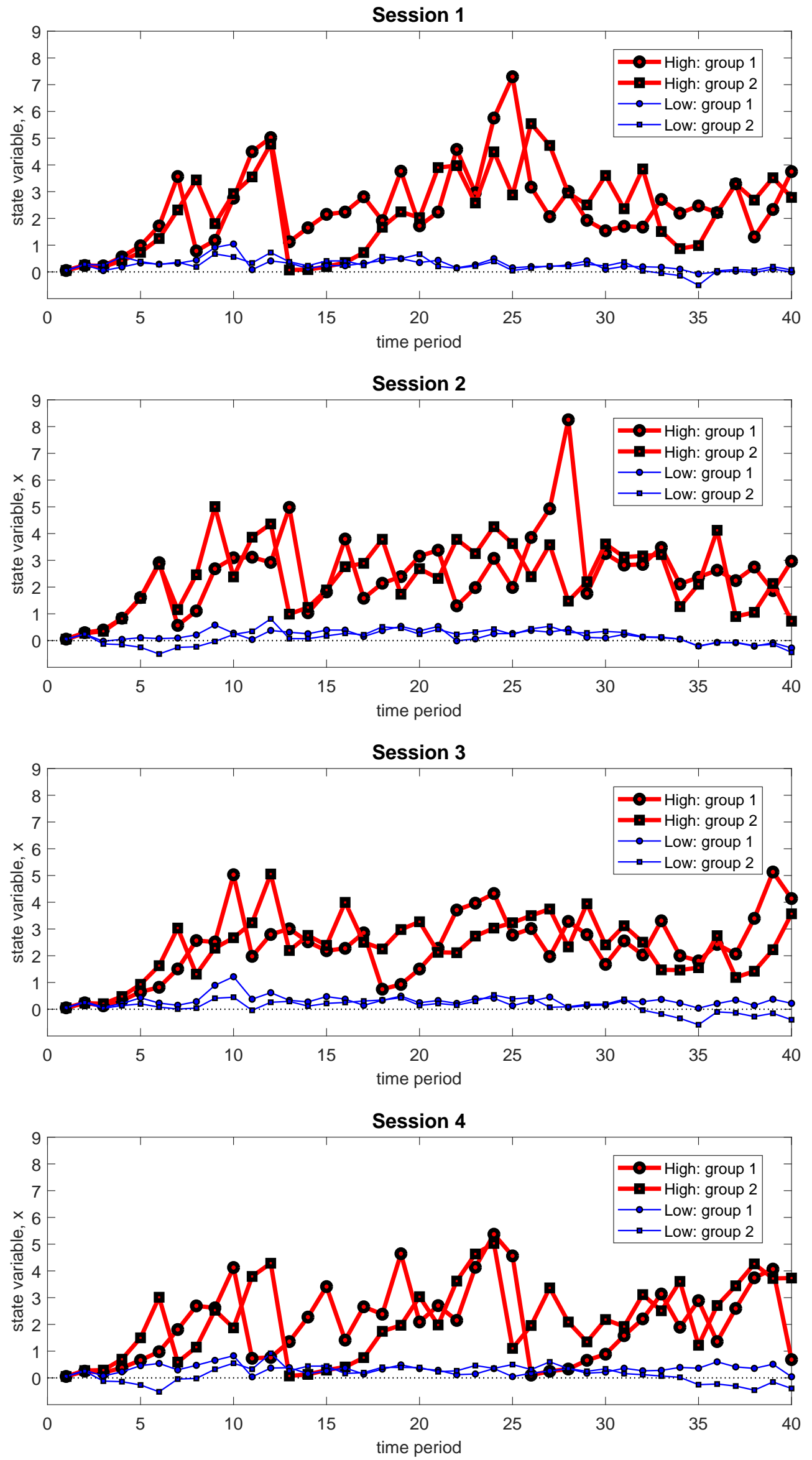

Figure 7: Time series of the state variable $x_{t}$ in all 16 groups. 
as well, and the standard deviation of these time series is significantly higher than they would be in a steady state of the model (where the standard deviation of $x_{t}$ is equal to that of $\epsilon_{t}$ which, by construction, is approximately 0.14). ${ }^{17}$

Summarizing our results thus far, we conclude that, when looking at the dynamics of both $n_{B, t}$ and $x_{t}$, Hypothesis 1 cannot be rejected, although, particularly for $n_{B, t}$, the difference between blocks is smaller than expected.

A more detailed look at the time series of $x_{t}$ leads to some interesting observations. First, the dynamics in the first half of the block are qualitatively similar for each of the eight High block groups. In each group the variable $x_{t}$ increases in several consecutive periods after which it 'crashes' in one period, sometimes by a considerable extent. ${ }^{18}$ After this crash $x_{t}$ starts to increase again. This cycle is repeated two or three times in each of the eight groups. Interestingly, this type of dynamics is characteristic for the type of heuristic switching model studied in the literature on heterogeneous agents, see the upper left panel of Fig. 3 (also see Fig. 2 in Brock and Hommes, 1998). However, in the second halves of the High blocks this structure in the dynamics disappears in each of the eight groups and the behavior of $x_{t}$ becomes more irregular with no apparent structure. Second, the dynamics of $x_{t}$ in the Low blocks are quite different and seem to be consistent with small irregular fluctuations around a fixed positive value, with a decrease in $x_{t}$ in the last couple of periods. ${ }^{19}$ Note that, since the non-zero steady state value $x^{+}$from Proposition 2.1 depends upon the behavioral parameters $\alpha$ and $\beta$, it is difficult to test directly whether the mean of $x_{t}$ equals $x^{+} \cdot{ }^{20}$

\footnotetext{
${ }^{17}$ We can reject the hypothesis of zero mean for $x_{t}$, both for the High and for the Low blocks at the $1 \%$ significance level, using the $t$-test ( $p$-values of 0.000 for both High and Low blocks). We can also reject the hypothesis that the variance of $x_{t}$ is equal to the variance of $\epsilon_{t}$, i.e., 0.02 , in favor of the alternative hypothesis that the variance is higher, both for the High and for the Low blocks at a $1 \%$ significance level, using the Chi-Square test ( $p$-values of 0.000 for both the High and Low blocks).

${ }^{18}$ Note that the initial value of $x$ was chosen to be $x_{0}=0$. It follows that in the first period of the experiment $x_{1}=\epsilon_{1}=0.0538$ (independent of the subjects' choices). If the first realization of the random variable $\epsilon_{t}$ would have been negative, it is likely that $x_{t}<0$ for all $t$.

${ }^{19}$ This decrease in the last couple of periods seems to be due, at least partially, to a large negative shock in period $t=35$ of $\epsilon_{35}=-0.2944$.

${ }^{20}$ Since subjects do not observe the realized values of the state variable $x_{t}$, and because $x_{t}$ only enters the profit functions (1) quadratically, its sign does not matter for the dynamics of fractions. Choosing $\lambda=-2.1$, instead of $\lambda=2.1$ would therefore lead to the same experimental results (abstracting from the effect of random shocks), but with the state variable alternating between positive and negative values. For the Low blocks the dynamics of the state variable will then resemble a noisy period-two cycle, instead of a noisy non-zero steady state.
} 


\begin{tabular}{|c|c|c|c|c|c|c|c|c|c|}
\hline & \multirow[b]{2}{*}{ Data } & \multicolumn{2}{|c|}{ IoC } & \multicolumn{2}{|c|}{ Predisposition } & \multicolumn{2}{|c|}{ Zero SS } & \multicolumn{2}{|c|}{ Non-Zero SS } \\
\hline & & Beta & S.E. & Alpha & S.E. & $\left(x^{*}, n^{*}\right)$ & $f^{\prime}(x *)$ & $\left(x^{+}, n^{+}\right)$ & $f^{\prime}\left(x^{+}\right)$ \\
\hline \multirow{9}{*}{ High } & Session 1. Group 1 & 0.08 & 0.01 & 0.31 & 0.11 & $(0,0.58)$ & 1.22 & $(2.30,0.48)$ & 0.57 \\
\hline & Session 1. Group 2 & 0.12 & 0.02 & 0.38 & 0.11 & $(0,0.64)$ & 1.34 & $(2.36,0.48)$ & 0.31 \\
\hline & Session 2. Group 1 & 0.12 & 0.02 & 0.35 & 0.11 & $(0,0.64)$ & 1.35 & $(2.42,0.48)$ & 0.28 \\
\hline & Session 2. Group 2 & 0.17 & 0.02 & 0.28 & 0.11 & $(0,0.74)$ & 1.56 & $(2.63,0.48)$ & -0.22 \\
\hline & Session 3. Group 1 & 0.09 & 0.02 & 0.13 & 0.11 & $(0,0.64)$ & 1.35 & $(2.76,0.48)$ & 0.29 \\
\hline & Session 3. Group 2 & 0.13 & 0.02 & 0.04 & 0.11 & $(0,0.72)$ & 1.52 & $(2.90,0.48)$ & -0.11 \\
\hline & Session 4. Group 1 & 0.13 & 0.02 & 0.19 & 0.12 & $(0,0.69)$ & 1.46 & $(2.69,0.48)$ & 0.05 \\
\hline & Session 4. Group 2 & 0.16 & 0.02 & 0.38 & 0.12 & $(0,0.71)$ & 1.49 & $(2.49,0.48)$ & -0.03 \\
\hline & All High groups & 0.12 & 0.01 & 0.25 & 0.04 & & & & \\
\hline \multirow{2}{*}{ High Long } & Session 5. Group 1 & 0.15 & 0.02 & 0.08 & 0.09 & $(0,0.75)$ & 1.58 & $(2.85,0.48)$ & -0.27 \\
\hline & Session 6. Group 2 & 0.16 & 0.02 & 0.10 & 0.09 & $(0,0.76)$ & 1.60 & $(2.82,0.48)$ & -0.32 \\
\hline \multirow{9}{*}{ Low } & Session 1. Group 1 & 3.35 & 0.84 & 0.26 & 0.11 & $(0,0.52)$ & 1.09 & $(2.23,0.48)$ & 0.82 \\
\hline & Session 1. Group 2 & 5.24 & 0.93 & 0.09 & 0.11 & $(0,0.61)$ & 1.27 & $(2.32,0.48)$ & 0.45 \\
\hline & Session 2. Group 1 & 11.35 & 1.66 & -0.17 & 0.13 & $(0,0.79)$ & 1.65 & $(2.35,0.48)$ & -0.46 \\
\hline & Session 2. Group 2 & 8.67 & 1.47 & 0.10 & 0.11 & $(0,0.68)$ & 1.43 & $(2.32,0.48)$ & 0.10 \\
\hline & Session 3. Group 1 & 2.36 & 0.67 & 0.26 & 0.10 & $(0,0.49)$ & 1.04 & $(2.18,0.48)$ & 0.92 \\
\hline & Session 3. Group 2 & 10.47 & 1.72 & -0.38 & 0.13 & $(0,0.81)$ & 1.69 & $(2.38,0.48)$ & -0.60 \\
\hline & Session 4. Group 1 & 5.04 & 1.04 & 0.16 & 0.11 & $(0,0.58)$ & 1.23 & $(2.29,0.48)$ & 0.54 \\
\hline & Session 4. Group 2 & 8.06 & 1.31 & 0.21 & 0.11 & $(0,0.65)$ & 1.36 & $(2.29,0.48)$ & 0.27 \\
\hline & All Low groups & 5.71 & 0.42 & 0.11 & 0.04 & & & & \\
\hline
\end{tabular}

Table 2: Estimation of discrete choice model with two parameters, the intensity of choice parameter, $\beta$, and the predisposition parameter, $\alpha$.

\subsection{Estimated discrete choice models}

Our next step is to fit the discrete choice model (4) to the experimental data. That is, we estimate the discrete choice model separately for the aggregate choices in each group. Table 2 shows the results. Columns 3 and 4 give the estimated values and the standard deviations for the intensity of choice parameter $\beta$, and columns 5 and 6 give the estimated values and the standard deviations for the predisposition parameter $\alpha$. We also estimated the model on the pooled data for all High block groups and all Low block groups.

The data for each of the sixteen groups can be described quite well by the discrete choice model, which confirms Hypothesis 2. In particular, the intensity of choice parameter $\beta$ is positive and significantly different from 0 for all groups. The predisposition parameter $\alpha$ is positive (implying a predisposition towards alternative $A$ ) and significantly different from 0 in five of the eight High block groups, but only significantly different from 0 in three of the eight Low blocks (and positive for only two of those). ${ }^{21}$

\footnotetext{
${ }^{21}$ Estimating the discrete choice model with the restriction $\alpha=0$ leads to new estimates for $\beta$, which are quite close to the estimates in Table 2.
} 
One remarkable feature of the parameter estimates stands out from Table 2. Although the estimated intensity of choice coefficients for the same type of blocks have roughly the same order of magnitude, these coefficients are radically different between different types of blocks, with the estimates for the Low block groups about 50 times as high as those for the High block groups. The subjects therefore seem to be much less sensitive to profit differences in the 'unstable' High blocks than in the 'stable' Low blocks. Clearly, this means that we have to reject Hypothesis 3. This is broadly consistent with the results of Anufriev, Bao, and Tuinstra (2016) who show that the estimated values of the intensity of choice increase when there is more structure in the time series of payoffs, and indeed the time series of payoffs in the Low blocks are less volatile and more predictable than those in the High blocks. ${ }^{22}$

For each group we superimposed the estimated values of $\alpha$ and $\beta$ in the stability graphs of Fig. 1. First consider the eight data points corresponding to the Low blocks (the right panel). As we expected, all of them are in the region of the parameter space where the zero steady state $\left(x^{*}, n^{*}\right)$ is unstable, but where the non-zero steady state $\left(x^{+}, n^{+}\right)$is locally stable. This is confirmed by the last four columns in Table 2, which show the zero and non-zero steady state and the slope of the dynamical system, at those steady states, respectively, that are implied by the estimated values of $\alpha$ and $\beta$. Comparing $x^{+}$with the mean value in the experiment, given in column 4 of Table 1, suggests that the dynamics indeed converges to the non-zero (positive) steady state in each of those groups, although there is some excess volatility around that steady state.

Now consider the estimated discrete choice models for the High block groups, which are depicted in the left panel of Fig. 1. When designing the experiment we expected the dynamics in these High blocks to be unstable, and indeed the variations in $x_{t}$ and, to a weaker extent, $n_{B, t}$, are higher in those blocks - see the discussion in Section 5.1. However, it turns out that the estimated parameters for these blocks are located in the region of local stability of the non-zero steady state, even if it requires very low levels of the intensity of choice parameter $\beta$. Moreover, the non-zero steady state values of $x^{+}$shown in Table 2, implied by the estimated values of $\alpha$ and $\beta$, are very close to the mean value of $x_{t}$ in each

\footnotetext{
${ }^{22}$ Another reason may be that the discrete choice model is not invariant with respect to changes in profit levels. That is, an increase in $\beta$ is equivalent with exactly the same increase in profits, see Eq. (4). However, in our experiment average profits, as well as average profit differences, are roughly the same for both types of blocks (although the standard deviation of profits is higher in the High blocks).
} 
of these eight High block groups. Although there is substantial volatility around the steady state, it seems that also in this case there is convergence to the nonzero steady state. Apparently, after experiencing the dynamics that is typical for the heuristic switching model in the first half of the block, subjects become more cautious and are able - at least to a certain extent - to stabilize the dynamics in this highly volatile and unstable environment, by behaving according to a discrete choice model with a low value of the intensity of choice parameter $\beta$.

\subsection{Further evidence on the endogeneity of the IoC pa- rameter}

The analysis in Sections 5.1 and 5.2 suggests that subjects in our experiment on the heuristic switching model have a tendency to adapt their choice behavior to their environment. Partly due to the time and space constraints imposed by using laboratory experiments, the number of subjects and periods in our experiment are limited to 10 subjects per group, and 40 periods per block, respectively. A relevant question is whether our results are robust to increases in the group size and time horizon. To investigate this, we run an additional treatment that differs from the two treatments described and analyzed above in three ways: (i) the parametrization of the underlying model is fixed over the course of the treatment, and equal to that of the High blocks in the other two treatments; (ii) the experiment runs for 60 periods; and (iii) we increase the group size to 33 participants. Note that the increase in the number of periods allows us to better study the adaptation of the intensity of choice parameter to the decision environment over time, and the increase in the number of participants gives us a less coarse approximation of the fraction $n_{B, t}$ of the original model. We ran two sessions of this treatment, with one group in each session.

The upper panels of Fig. 8 show the evolution of the fraction $n_{B, t}$ in the two groups. The larger group size hardly smooths out the dynamics: The fractions are still quite volatile over the full 60 periods of the experiment. In fact, in quite some periods at least around half of the subjects switch to another heuristic. However, the larger group size contributes to the fact that the fraction $n_{B, t}$ rarely approaches its boundary values of 0 and 1 closely - only in one period in session 2 all 33 participants choose the same heuristic. The middle panels of Fig. 8 show the dynamics of the state variable $x_{t}$ for the two groups. Again, both groups start 

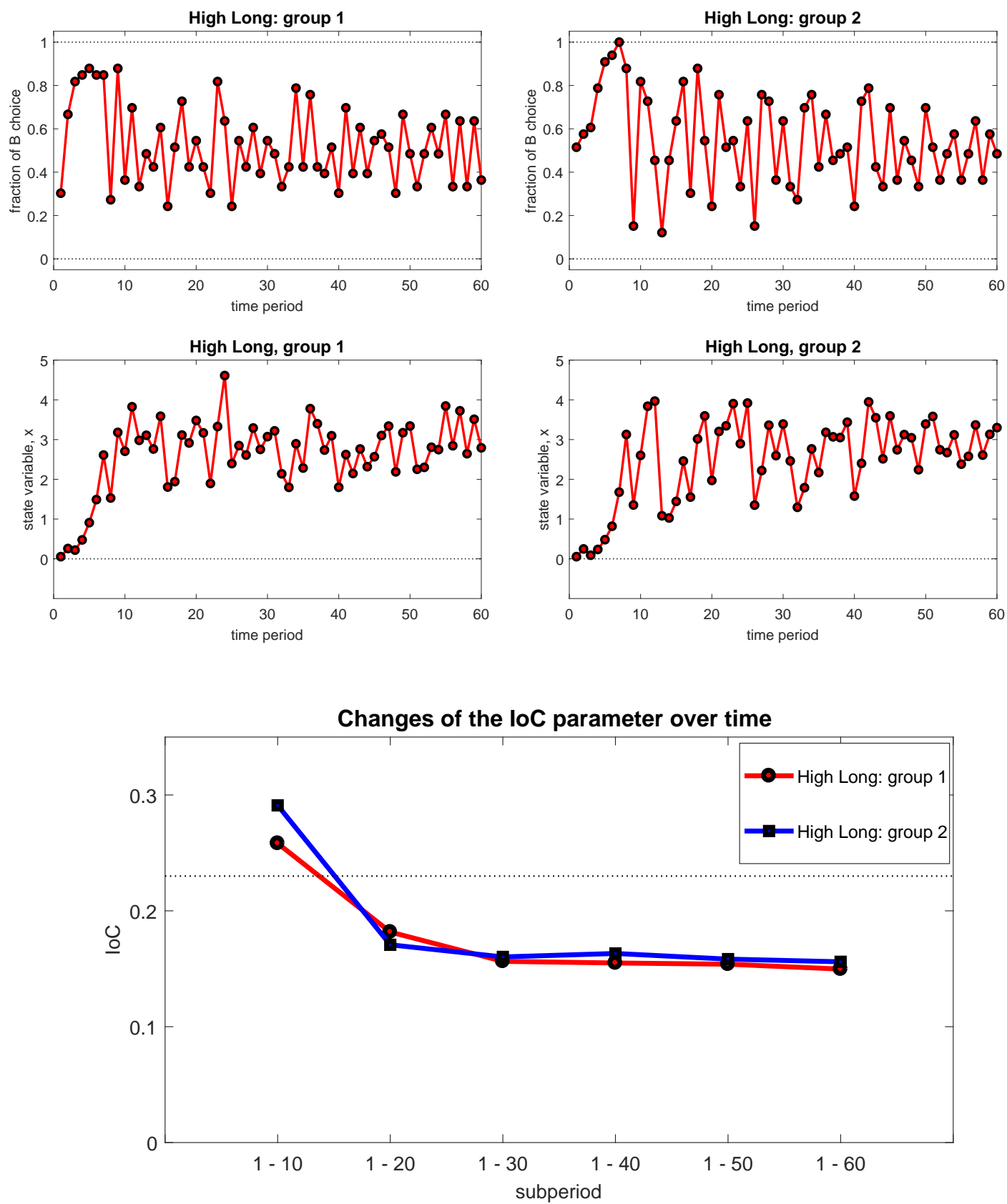

Figure 8: Experimental results of the two groups in the additional High Long treatment. Upper panels: Dynamics of fractions of $B$-choices, $n_{B}$. Left lower panel: Dynamics of the state variable, $x$. Right lower panel: Changes in the intensity of choice parameter, estimated on the 6 extending subsamples of the data from the High Long treatment. The dotted line represents the boundary above which the non-zero steady state is unstable. 
out with the familiar pattern of a slowly increasing bubble which crashes after a couple of periods, after which the state variable increases again. Eventually - as with the shorter High blocks studied above - the dynamics fluctuate in an erratic manner around some positive fixed value of the state variable (note that the amplitude of the fluctuations in the state variable is less than that in the High blocks, again due to the increased group size). These results suggest that our earlier findings for the High cost blocks are robust to increasing the number of periods and the group size. This is confirmed by the descriptive statistics for this High Long treatment in Table 1 and the estimation of the discrete choice model for this treatment presented in Table 2 and in Fig. 1, which are consistent with those for the High blocks.

The larger number of periods in the High Long treatment allows us to investigate in more depth how subjects adapt their behavior to the dynamics of payoffs. To that end, we split our sample into six subsamples of increasing length, with the first subsample consisting of the first 10 time periods, the second subsample consisting of the first 20 time periods, and the last 'subsample' corresponding to the full time series of 60 periods. The discrete choice model is estimated on each of these six subsamples, and the estimated value of the intensity of choice parameter $\beta$ is presented in the bottom panel of Fig. 8. We see that the estimated intensity of choice parameter indeed decreases over time. This parameter lies above the stability threshold (assuming $\alpha=0$ ) of the non-zero steady state for the first subsample, but below it from the second subsample onwards. Moreover, the estimated values show very similar patterns for the two different groups.

Why do subjects become less sensitive to past performance over time in the High cost environment? This may be because in that environment payoffs are very erratic and volatile, and may not predict future payoffs very well. Subjects may realize this after the first ten to fifteen periods, and then start to rely to a lesser extent upon past performance when choosing their heuristic. This in turn brings down volatility. Indeed, the standard deviation of profit differences is equal to 4.76 and 5.21 in the first 20 periods in the two High Long groups, but for periods 21-40 the standard deviation is down to 4.25 and 4.13 , respectively, and it decreases to 3.01 and 2.95 for the last 20 periods. 


\section{Conclusion}

After its introduction in Brock and Hommes (1997, 1998), the heuristic switching model has become a workhorse model in the field of heterogeneous agents and agent-based modeling. The standard discrete choice framework, according to which agents choose between heuristics - typically corresponding to different forecasting rules - on the basis of their past performance, is a central element of this model. The resulting changes in the distribution of agents over heuristics influence the evolution of the state variable (typically, the asset price), which feeds back into the performance of the heuristics. This interaction between the dynamics of the state variable, and the dynamics of the distribution of heuristics, is capable of generating endogenous bubbles and crashes, excess volatility, and other stylized facts of financial markets. It therefore presents a natural extension to the work on the dynamics arising from the coexistence of fundamental and trend-following rules that started with Day and Huang (1990) and Chiarella (1992).

In this paper we present a laboratory experiment to test the heuristic switching model. Similar to the experiment described in Anufriev, Bao, and Tuinstra (2016), the only task of the subjects is to choose one of two heuristics, and subjects are paid according to the performance of the heuristic they choose. Contrary to the previous experiment, however, in the experiment presented here the subjects' aggregate choices determine, through a hidden state variable, the payoffs generated by the heuristics - an important feature of the standard heuristic switching model as well. In particular, our experiment reproduces, in a stylized form, the interaction between a costly sophisticated and a simple cheap heuristic, where the latter is destabilizing when used by many subjects, and the former is stabilizing.

We vary the (implicit) cost of using the stabilizing heuristic between different blocks in the experiment. Theoretically, the heuristic switching model will generate dynamics that are relatively stable when costs are low (in the Low blocks) and give rise to endogenous bubbles and crashes when costs are high (in the High blocks). Although the theoretical prediction is confirmed in the Low blocks, the results from the High blocks are ambiguous. In particular, we do observe endogenous bubbles and crashes in the first half of the High blocks. However, this characteristic pattern disappears in the second half of the High blocks, and the dynamics become more stable. We estimate the discrete choice model on the experimental data and find that the intensity of choice parameter is much lower 
for the High blocks, suggesting that subjects adapt their behavior and become less sensitive to payoff differences in a less stable environment - this is consistent with the findings in Anufriev, Bao, and Tuinstra (2016). The reason that subjects adapt their behavior might be driven by the fact that, in the high cost environment, payoff differences tend to be highly volatile and unpredictable, and therefore may not perform well as a predictor of future success. Upon realizing this, subjects' response to past payoffs becomes weaker, which brings down this volatility in payoff differences endogenously. This is confirmed in the High Long treatment, which features more decision periods. Here the estimated values of the intensity of choice parameter indeed decrease over time, inducing a reduction in volatility. An interesting extension for future research would be a treatment with a fixed group of subjects, each of whom has to choose between the two alternatives again, but where - without informing the subjects directly - the costs associated with alternative $A$ change at several instances during the experiment. Such a change in costs will effect the volatility of payoff differences, which may lead subjects to adapt their behavior. ${ }^{23}$ A practical difficulty of running such a treatment is that it requires many decision periods and will therefore take a long time, with the risk that boredom or lack of concentration on the part of the subjects will effect the results. Nevertheless, based upon our High-Low and Low-High treatments, where such a change in costs was implemented, but accompanied by a reshuffling of the groups and a restart of the time series of payoffs, we conjecture that subjects will respond to volatility of profit differences and the estimated intensity of choice parameter will change over time.

Our results have important implications for the way choice behavior is modeled in heuristic switching models. They suggest that a model in which the intensity of choice parameter is endogenous, and depends positively upon some measure of volatility of payoff differences, potentially provides a better description of choice behavior. Adapting the benchmark model in this way may turn out to be quite relevant since the assumption that the intensity of choice parameter is exogenously given can impose a bias in the conclusions derived from theoretical heuristic switching models. Consider, for example, a volatile financial market that is described well by a particular heuristic switching model. On the basis of that model the financial regulator may want to implement a policy that - based upon numerical

\footnotetext{
${ }^{23}$ Recall that in our experiment subjects do not observe the costs for the stabilizing heuristic directly, nor do they observe the evolution of the state variable $x_{t}$. That is, their choice has to be solely based on past payoffs.
} 
simulations - stabilizes market dynamics. However, if traders react to the increased stability and predictability of profits in this market by starting to respond more strongly to profit differences - as suggested by our experimental results this may strongly mitigate the effect of the policy. 


\section{References}

Allen, H., And M. P. Taylor (1990): "Charts, Noise and Fundamentals in the London Foreign Exchange Market," The Economic Journal, 100, 49-59.

Anufriev, M., T. BaO, And J. Tuinstra (2016): "Microfoundations for switching behavior in heterogeneous agent models: An experiment," Journal of Economic Behavior and Organization, 129, 74-99.

Anufriev, M., and C. Hommes (2012a): "Evolution of Market heuristics," The Knowledge Engineering Review, 27(2), 255-271.

(2012b): "Evolutionary Selection of Individual Expectations and Aggregate Outcomes in Asset Pricing Experiments," American Economic Journal: Microeconomics, 4(4), 35-64.

Anufriev, M., and V. Panchenko (2009): "Asset prices, traders behavior and market design," Journal of Economic Dynamics and Control, 33(5), 1073-1090.

Anufriev, M., And J. Tuinstra (2013): "The impact of short-selling constraints on financial market stability in a heterogeneous agents model," Journal of Economic Dynamics and Control, 37(8), 1523-1543.

Boswijk, H. P., C. H. Hommes, and S. Manzan (2007): "Behavioral heterogeneity in stock prices," Journal of Economic Dynamics and Control, 31, 1938-1970.

Branch, W. A. (2004): "The Theory of Rationally Heterogeneous Expectations: Evidence from Survey Data on Inflation Expectations," The Economic Journal, 114(497), 592-621.

Branch, W. A., And G. W. Evans (2006): "Intrinsic heterogeneity in expectation formation," Journal of Economic theory, 127(1), 264-295.

Brock, W. A., And C. H. Hommes (1997): "A Rational Route to Randomness," Econometrica, 65(5), 1059-1095.

(1998): "Heterogeneous beliefs and routes to chaos in a simple asset pricing model," Journal of Economic Dynamics and Control, 22(8), 1235-1274.

Brock, W. A., C. H. Hommes, and F. O. Wagener (2005): "Evolutionary dynamics in markets with many trader types," Journal of Mathematical Economics, 41(1), 7-42. 
Brock, W. A., C. H. Hommes, and F. O. O. Wagener (2009): "More hedging instruments may destabilize markets," Journal of Economic Dynamics and Control, 33(11), 1912-1928.

Chiarella, C. (1992): "The dynamics of speculative behaviour," Annals of operations research, 37(1), 101-123.

Chiarella, C., R. Dieci, and L. Gardini (2001): "Asset price dynamics in a financial market with fundamentalists and chartists," Discrete Dynamics in Nature and Society, 6(2), 69-99.

(2006): "Asset price and wealth dynamics in a financial market with heterogeneous agents," Journal of Economic Dynamics and Control, 30(9), 17551786.

Chiarella, C., and X.-Z. He (2002): "Heterogeneous Beliefs, Risk and Learning in a Simple Asset Pricing Model," Computational Economics, 19, 95-132.

- (2003): "Dynamics of beliefs and learning under $a_{L}$-processes - the heterogeneous case," Journal of Economic Dynamics and Control, 27, 503-531.

Chiarella, C., X.-Z. He, and C. Hommes (2006): "A dynamic analysis of moving average rules," Journal of Economic Dynamics and Control, 30, 17291753.

Chiarella, C., X.-Z. He, and R. C. Zwinkels (2014): "Heterogeneous expectations in asset pricing:Empirical evidence from the S\&P500," Journal of Economic Behavior and Organization, 105, 1-16.

Cornea-Madeira, A., C. Hommes, and D. Massaro (2017): "Behavioral heterogeneity in US inflation dynamics," Journal of Business and Economic Statistics, forthcoming.

DAY, R. H., And W. HuAng (1990): "Bulls, bears and market sheep," Journal of Economic Behavior and Organization, 14, 299-219.

Dindo, P., And J. Tuinstra (2011): "A class of evolutionary models for participation games with negative feedback," Computational Economics, 37(3), 267300.

EzeKIEL, M. (1938): "The cobweb theorem," The Quarterly Journal of Economics, 52(2), 255-280. 
FischBACHER, U. (2007): "z-Tree: Zurich toolbox for ready-made economic experiments," Experimental Economics, 10(2), 171-178.

Frankel, J. A., and K. A. Froot (1990): "Chartists, Fundamentalists, and Trading in the Foreign Exchange Market," The American Economic Review, 80(2), 181-185.

Gaunersdorfer, A. (2000): "Endogenous fluctuations in a simple asset pricing model with heterogeneous agents," Journal of Economic Dynamics and Control, 24(5), 799-831.

Gaunersdorfer, A., C. H. Hommes, and F. O. Wagener (2008): "Bifurcation routes to volatility clustering under evolutionary learning," Journal of Economic Behavior \& Organization, 67(1), 27-47.

Goldbaum, D., And B. Mizrach (2008): "Estimating the Intensity of Choice in a Dynamic Mutual Fund Allocation Decision," Journal of Economic Dynamics and Control, 32, 3866-3876.

Goldbaum, D., and R. C. Zwinkels (2014): "An empirical examination of heterogeneity and switching in foreign exchange markets," Journal of Economic Behavior and Organization, 107(2), 667-684.

Greiner, B. (2015): "Subject Pool Recruitment Procedures: Organizing Experiments with ORSEE," Journal of the Economic Science Association, 1(1), $114-125$.

Heemeijer, P., C. Hommes, J. Sonnemans, and J. Tuinstra (2009): "Price stability and volatility in markets with positive and negative expectations feedback: An experimental investigation," Journal of Economic Dynamics and Control, 33, $1052-1072$.

HEY, J. D. (1994): "Expectations formation: Rational or adaptive or?," Journal of Economic Behavior and Organization, 25(3), 329-349.

Hommes, C. (2005): "Heterogeneous agent models: two simple examples," in Nonlinear Dynamical Systems in Economics, pp. 131-164. Springer.

(2011): "The heterogeneous expectations hypothesis: Some evidence from the lab," Journal of Economic Dynamics and Control, 35, 1-24. 
(2013): Behavioral Rationality and Heterogeneous Expectations in Complex Economic Systems. Cambridge University Press.

Hommes, C., J. Sonnemans, J. Tuinstra, and H. van de Velden (2005): "Coordination of expectations in asset pricing experiments," The Review of Financial Studies, 18(3), 955-980.

(2007): "Learning in cobweb experiments," Macroeconomic Dynamics, 11(1), 8-33.

Kelley, H., And D. Friedman (2002): "Learning to forecast price," Economic Inquiry, 40(4), 556-573.

Panchenko, V., S. Gerasymchuk, and O. V. Pavlov (2013): "Asset price dynamics with heterogeneous beliefs and local network interactions," Journal of Economic Dynamics and Control, 37(12), 2623-2642.

Schmalensee, R. (1976): "An experimental study of expectation formation," Econometrica, 44(1), 17-41.

Schmitt, N., J. Tuinstra, And F. Westerhoff (2017): "Side effects of nonlinear profit taxes in an evolutionary market entry model: abrupt changes, coexisting attractors and hysteresis problems," Journal of Economic Behavior \& Organization, 135, 15-38.

ter Ellen, S., C. Hommes, And R. Zwinkels (2017): "Comparing Behavioural Heterogeneity Across Asset Classes," Discussion paper, SSRN paper.

Tuinstra, J., M. Wegener, and F. Westerhoff (2014): "Positive welfare effects of trade barriers in a dynamic partial equilibrium model," Journal of Economic Dynamics and Control, 48, 246-264.

Westerhoff, F. H., And R. Dieci (2006): "The effectiveness of Keynes-Tobin transaction taxes when heterogeneous agents can trade in different markets: a behavioral finance approach," Journal of Economic Dynamics and Control, 30(2), 293-322. 


\section{APPENDIX}

\section{A Stylized HSM}

In the experiment we use a stylized heuristic switching model. Below we provide a formal analysis of this model and show that the properties of the original BrockHommes model are preserved.

The dynamics of the stylized model are given by the $1 \mathrm{D}$ equation (5). We switch the noise off, $\epsilon_{t}=0$, to focus on the analysis of the deterministic skeleton $x_{t+1}=f\left(x_{t}\right)$, with

$$
f(x)=\frac{\lambda x}{1+\exp \left[\alpha+\beta\left(x^{2}-C\right)\right]},
$$

where $C=W_{B}-W_{A}$ can be interpreted as the costs for alternative $A$. Given the value of the state variable, $x_{t}$, we can easily recover the fraction of $B$-choices as

$$
n_{B, t+1}=\frac{1}{1+\exp \left[\alpha+\beta\left(x_{t}^{2}-C\right)\right]}=\frac{x_{t+1}}{\lambda x_{t}} .
$$

In the analysis below we assume $C>0$ and $\beta \geq 0$, but we will not restrict the other parameters. Note that in the experiment presented in the paper we set $\lambda=2.1$ and $C=8$ (High blocks) or $C=0.1$ (Low blocks). The behavioral parameters $\alpha$ and $\beta$, from the discrete choice model, describe subjects' choices.

The following result characterizes the dynamic properties of (A.1).

Proposition A.1. Let $f$ be defined in (A.1) with $C>0$ and $\beta \geq 0$. Consider the system $x_{t+1}=f\left(x_{t}\right)$ and let $n^{*}=1 /(1+\exp [\alpha-\beta C])$.

1. $x^{*}=0$ is a steady state for all parameter values. In this steady state the fraction of agents choosing alternative $B$ is given by $n_{B}^{*}=n^{*}$. This steady state is unique for $\lambda<1$ and globally stable when $\left|\lambda n^{*}\right|<1$.

2. For $\lambda>0$, the system undergoes a pitchfork bifurcation when $\lambda=1 / n^{*}>1$. At the bifurcation two non-zero steady states, $x^{+}>0$ and $x^{-}=-x^{+}$, are created, with corresponding steady state fraction $n_{B}^{+}=n_{B}^{-}=1 / \lambda$.

3. For $\lambda<0$, a period doubling bifurcation occurs when $\lambda=-1 / n^{*}<-1$. At the bifurcation a two-cycle $\left\{x^{+}, x^{-}\right\}$, with $x^{+}>0$ and $x^{-}=-x^{+}$, is created, with corresponding steady state fraction $n_{B}^{+}=n_{B}^{-}=-1 / \lambda$.

Proof. It is obvious that $x^{*}=0$ is a steady state of the system. Other steady states are solutions of the equation

$$
1=\frac{\lambda}{1+\exp \left[\alpha+\beta\left(x^{2}-C\right)\right]} \quad \Leftrightarrow \quad \exp \left[\alpha+\beta\left(x^{2}-C\right)\right]=\lambda-1 .
$$


This equation has no solutions when $\lambda \leq 1$. For the case $\lambda>1$ we find that

$$
x^{2}=\frac{\ln (\lambda-1)-\alpha}{\beta}+C .
$$

Therefore, for $\alpha<\ln (\lambda-1)+\beta C$ (which is equivalent to $\lambda>1 / n^{*}$ ) the original system has two non-zero steady states

$$
x^{ \pm}= \pm \sqrt{\frac{\ln (\lambda-1)-\alpha}{\beta}+C} .
$$

In both steady states, $n_{B}^{*}$, the fraction of agents choosing $B$ is equal to $1 / \lambda$.

To investigate the local stability properties of the steady states we take the derivative of $f(x)$ :

$$
f^{\prime}(x)=\frac{\lambda}{1+\exp \left[\alpha+\beta\left(x^{2}-C\right)\right]}-2 x^{2} \lambda \beta \frac{\exp \left[\alpha+\beta\left(x^{2}-C\right)\right]}{\left(1+\exp \left[\alpha+\beta\left(x^{2}-C\right)\right]\right)^{2}} .
$$

In the zero steady state the slope is $\lambda /(1+\exp [\alpha-\beta C])=\lambda n^{*}$. Thus it is locally asymptotically stable for $\left|\lambda n^{*}\right|<1$. Moreover, we can also write

$$
\left|x_{t+1}\right|=\left|\lambda x_{t}\right| \frac{1}{1+\exp \left[\alpha+\beta\left(x^{2}-C\right)\right]} \leq\left|\lambda x_{t}\right| \frac{1}{1+\exp [\alpha-\beta C]}=|\lambda|\left|x_{t}\right| n^{*} .
$$

Therefore, when $\left|\lambda n^{*}\right|<1$ the map is a contraction and the zero steady state is globally stable.

The slope at the zero steady state is equal to 1 when $\lambda=1 / n^{*}$. Given that the map $f$ is odd, system is symmetric with respect to $x$. Therefore at $\lambda=1 / n^{*}$ the system undergoes a pitchfork bifurcation. We have shown above that the two steady states for $\lambda>1 / n^{*}$ are $x^{+}$and $x^{-}$as given by (A.3).

When $\lambda=-1 / n^{*}$, the slope at the zero steady state is equal to -1 and, hence, the system undergoes a period-doubling bifurcation. For $\lambda<-1 / n^{*}$ we can define $x^{+}$and $x^{-}$in analogy with (A.3) as

$$
x^{ \pm}= \pm \sqrt{\frac{\ln (|\lambda|-1)-\alpha}{\beta}+C} .
$$

Then

$$
f\left(x^{+}\right)=\frac{\lambda x^{+}}{1+\exp \left[\alpha+\beta\left(\left(x^{+}\right)^{2}-C\right)\right]}=\frac{\lambda x^{+}}{|\lambda|}=-x^{+}=x^{-} \quad \text { and } \quad f\left(x^{-}\right)=x^{+} .
$$

Thus, $\left\{x^{+}, x^{-}\right\}$is a two-cycle created at the moment of the bifurcation. It then follows from (A.2) that along this cycle $n_{B} \equiv-1 / \lambda$.

We illustrate the properties of the system in Fig. 9 with six bifurcation di- 

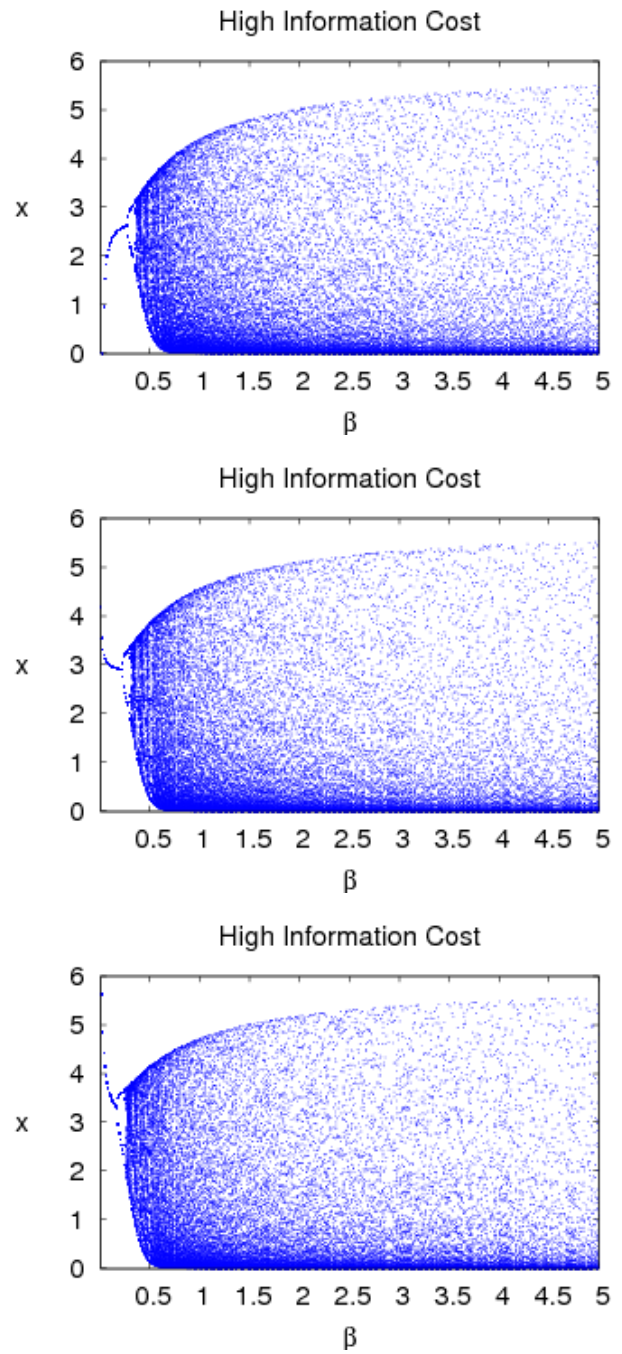
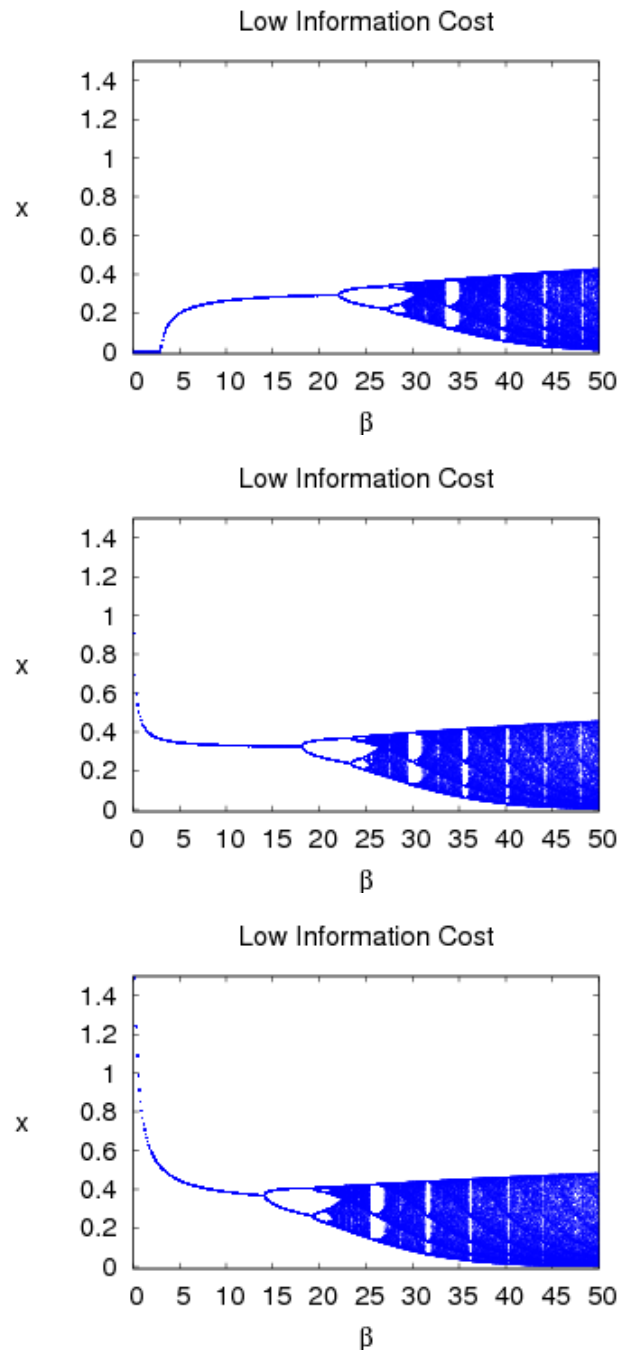

Figure 9: Bifurcation diagrams with respect to the Intensity of Choice, $\beta$, for system (A.1) with $\lambda=2.1$. Left panels: High cost, $C=8$. Right panels: Low cost, $C=0.1$. Predisposition parameter: $\alpha=0.4$ (top panels), $\alpha=0$ (middle panels) and $\alpha=-0.4$ (lower panels).

agrams. To build them we fix $\lambda=2.1$ and consider two values of cost, as in the experiment. The diagrams in the left panels are built for High cost $(C=8)$ and the diagrams in the right panels are built for Low cost $(C=0.1)$. For each diagram we fix a specific value of $\alpha$ as follows: $\alpha=0.4$ for the diagrams in the top panels, $\alpha=0$ for the diagrams in the middle panels, and $\alpha=-0.4$ for the diagrams in the lower panels. Finally, for each diagram, we vary $\beta$ between 0 and 5 for High cost and between 0 and 50 for Low cost, and show (against every value of $\beta$ ) 500 points of the dynamics of system (A.1), initialized at $x_{0}=0.25$, after 400 transitory periods. ${ }^{24}$

We note that the left and right diagrams look similar for every row, exhibiting

\footnotetext{
${ }^{24}$ Since the noise $\epsilon_{t}$ is off, the state variable $x$ will stay positive on every trajectory for such initial value. The bifurcation diagrams for negative initial value look similar to those shown in Fig. 9, but it is in the negative domain of the state variable.
} 
the typical shape of the Brock-Hommes framework. When $\beta$ increases, first, the zero steady state loses stability through the pitchfork bifurcation, as proven in Proposition A.1.2. Two non-zero steady states are created, and we observe one of them, $x^{+}$. It is straightforward to check that the slope of $f$ in each of the steady states is given by

$$
f^{\prime}\left(x^{ \pm}\right)=1-2 \beta\left(\frac{\ln (\lambda-1)-\alpha}{\beta}+C\right)\left(1-\frac{1}{\lambda}\right) .
$$

Therefore, for any value of $\lambda$ and the other parameters, when $\beta$ increases the slope will decrease and the non-zero steady state will eventually lose stability through a period doubling bifurcation. A further increase in $\beta$, as shown in Fig. 9, leads to complicated dynamics with increasing ranges of the state variable. The points of bifurcations occur much earlier, however, for the High cost case than for the Low cost case. Bifurcations diagrams do not change significantly also with changes in $\alpha$ (except that the zero steady state is never stable for relatively high $\alpha$, but it is stable for low values of $\beta$, when $\alpha$ is low).

Our choice of parameter values in the experiment was motivated by these diagrams, as they predict different behavior in two treatments for very large range of values of behavioral parameters, $\beta$ and $\alpha$, under assumption of similar behavior in two treatments. 


\section{B Experimental Instructions}

Below we provide the short version of the instructions. For the full version which includes the examples of screens explained to the participants, quiz, and questionnaire, see the On-line Appendix.

\section{General information}

Today you will participate in an experiment which will require you to make economic decisions. During the experiment you will be able to earn a number of points. The better your decisions are, the more points you are likely to earn. These points will be converted into Australian dollars after the experiment.

\section{Task overview}

Consider a situation in which you are offered a choice between two investment alternatives. As in real markets, the profits of these alternatives depend on the decisions of all market participants (including yourself) and also on chance.

This experiment has two parts ${ }^{25}$, each consisting of $\mathbf{4 0}$ decision periods. In the beginning of each part you will be randomly matched to several other participants in this experiment that will be active in the same market. The same participants will be in the same market as you during this first part of the experiment. Every decision period you will have to choose one of the two alternatives, A or B. After you, and all other participants, made a decision, the profits of both alternatives are determined. Your decision will determine how many points you may get for this period. This number of points will be used to determine your final earnings. The exact procedure that determines your final earnings from the experiment is explained below (under Remuneration).

Example: if in some decision period you have chosen $\mathbf{A}$, and then (on the basis of this decision and others' choices) the profits of the alternatives turned out to be 7.35 for $\mathbf{A}$ and 2.53 for $\mathbf{B}$, then for this period you will be able to get 7.35 points.

Before you select one of the alternatives you will be shown the profits of both alternatives for all previous periods, as well as the history of your own decisions. The only exception is the very first period (for each of the two parts), when no history is available. Note that the prospective profits are not known to you at the moment of your decision. After you made your decision for a given period, you will get all the information on realized profits of both alternatives as well as your number of points for this period.

After the first part of the experiment is finished, i.e., after the first 40 decision

\footnotetext{
${ }^{25}$ In additional sessions with High Long block Instructions were changed accordingly.
} 
periods, the second part of the experiment starts. For this part you will be assigned to another group with several other participants (some, but not all, of them may be from the market that you participated in in the first part). This new group of participants now participates in another market, which is similar but where profits of the two alternatives are determined in a different manner. You will perform the same task of choosing between the two alternatives for another 40 periods.

You will see a countdown timer at every decision period. You do not have to finalize your decision in that time interval, but we nevertheless ask you to make your decisions in a timely manner.

\section{Remuneration}

Your total earnings in this experiment will be determined after the experiment by the following procedure. Two different periods will be chosen randomly by the computer program from the 40 periods of the first part of the experiment. Another two periods will also be chosen randomly by the computer program from the 40 periods of the second part of the experiment.

The sum of your points from these 4 periods divided by 2 will constitute the final earnings in Australian Dollars. Your final earnings will be rounded to the nearest value with a half-dollar precision.

In addition you will receive 10 AUD as show-up fee.

Note that with this procedure your performance for every period of the experiment potentially matters for your final monetary payoff.

At the end of each part of the experiment you will be shown the final screen with information about all your decisions in this part as well as profits of the two alternatives. You will also be informed which decision periods were randomly chosen for payment purposes. If you wish, you can keep this information for yourself in the last page of these Instructions.

\section{Additional information}

- Before the experiment starts you will have to take a short quiz which is designed to check your understanding of the instructions.

- By the end of the experiment you will be asked to answer a questionnaire. Inserted data will be processed in nameless form only. Please fill in the correct information.

- During the experiment any communication with other participants, whether verbal or written, is forbidden. The use of phones, tablets or any other 
gadgets is not allowed. Violation of the rules can result in exclusion from the experiment without any remuneration.

- Please follow the instructions carefully at all the stages of the experiment. If you have any questions or encounter any problems during the experiment please raise your hand and the experimenter will come to help you.

Please ask any question you have now! 